\title{
Weighted Completion of Galois Groups and Galois Actions on the Fundamental Group of $\mathbb{P}^{1}-\{0,1, \infty\}$
}

\author{
RICHARD HAIN $^{1}$ and MAKOTO MATSUMOTO ${ }^{2}$ \\ ${ }^{1}$ Department of Mathematics, Duke University, Box 90320, Durham, NC 27708-0320, U.S.A. \\ e-mail:hain@math.duke.edu \\ ${ }^{2}$ Department of Mathematics, Faculty of Science, Hiroshima University, Higashi-Hiroshima, \\ 739-8526 Japan.e-mail: m-mat@math.sci.hiroshima-u.ac.jp
}

(Received: 5 November 2001; accepted in final form: 28 January 2003)

\begin{abstract}
Fix a prime number $\ell$. We prove a conjecture stated by Ihara, which he attributes to Deligne, about the action of the absolute Galois group on the pro- $\ell$ completion of the fundamental group of the thrice punctured projective line. Similar techniques are also used to prove part of a conjecture of Goncharov, also about the action of the absolute Galois group on the fundamental group of the thrice punctured projective line. The main technical tool is the weighted completion of a profinite group with respect to a reductive representation (and other appropriate data).
\end{abstract}

Mathematics Subject Classifications. Primary 14F35, 14G32; Secondary 11G55, 11R34, 14F42, 14H30.

Key words. Galois cohomology, Galois group, mixed Tate motive, Tannakian category, weighted completion.

\section{Introduction}

Fix a prime number $\ell$. In this paper we prove a conjecture [16, p. 300], which Ihara attributes to Deligne, about the action of the absolute Galois group on the pro- $\ell$ completion of the fundamental group of the thrice punctured projective line. It is stated below. Similar techniques are also used to prove part of a conjecture of Goncharov [11, Conj. 2.1], also about the action of the absolute Galois group on the fundamental group of the thrice punctured projective line, and which derives from the conjectures of Deligne and Ihara and questions of Drinfeld [7, p. 859].

Ihara's version of Deligne's conjecture concerns the outer action

$$
\phi_{\ell}: G_{Q} \rightarrow \text { Out } \pi_{1}\left(\mathbb{P}^{1}(\mathbb{C})-\{0,1, \infty\}, x\right)^{(\ell)}
$$

of the absolute Galois group $G_{Q}$ on the pro- $\ell$ fundamental group of the thrice punctured projective line. The pro- $\ell$ completion of $\pi_{1}\left(\mathbb{P}^{1}(\mathbb{C})-\{0,1, \infty\}, x\right)$ is the inverse limit of its finite quotients of $\ell$-power order. We shall denote it by $\pi^{(\ell)}$. 
Ihara defines a filtration

$$
G_{\mathrm{Q}}=I_{\ell}^{0} G_{\mathrm{Q}} \supseteq I_{\ell}^{1} G_{\mathrm{Q}} \supseteq I_{\ell}{ }^{2} G_{\mathrm{Q}} \supseteq \cdots
$$

of $G_{\mathrm{Q}}$ by

$$
I_{\ell}{ }^{m} G_{\mathrm{Q}}=\operatorname{ker}\left\{\phi_{\ell}: G_{\mathrm{Q}} \rightarrow \operatorname{Out}\left(\pi^{(\ell)} / L^{m+1} \pi^{(\ell)}\right)\right\}
$$

where $L^{m} \pi^{(\ell)}$ denotes the $m$ th term of the lower central series of $\pi^{(\ell)}$ indexed so that $L^{1} \pi^{(\ell)}=\pi^{(\ell)}$. It is independent of the choice of base point $x$ but depends on the choice of the prime $\ell$, as the $\ell$-adic cyclotomic character induces an isomorphism $\mathrm{Gr}_{I_{\ell}}^{0} G_{\mathrm{Q}} \cong Z_{\ell}^{\times}$. It has the property that

$$
\left[I_{\ell} 2^{m} G_{\mathrm{Q}}, I_{\ell}{ }^{n} G_{\mathrm{Q}}\right] \subseteq I_{\ell}^{m+n} G_{\mathrm{Q}}
$$

from which it follows that the positive part

$$
\mathrm{Gr}_{I_{\ell}}^{>0} G_{\mathrm{Q}}:=\bigoplus_{n>0} \mathrm{Gr}_{I_{\ell}}^{n} G_{\mathrm{Q}}
$$

of the associated graded group of $G_{\mathrm{Q}}$ is a Lie algebra over $Z_{\ell}$ with bracket induced by the group commutator.

In this paper we shall prove:

CONJECTURE 1 ([16, p. 300]). The $Q_{\ell}$-form $\left(\mathrm{Gr}_{I_{\ell}}^{>0} G_{\mathrm{Q}}\right) \otimes Q_{\ell}$ of the positive part of $\mathrm{Gr}_{I_{\ell}}^{\bullet} G_{\mathrm{Q}}$ is generated as a Lie algebra by elements $s_{3}, s_{5}, s_{7}, \ldots$ where $s_{2 n+1} \in$ $\mathrm{Gr}_{I_{\ell}}^{2 n+1} G_{\mathrm{Q}}$.

In fact, combining this result with the work of Ihara [16], one can see (cf. Remark 8.6) that there is a $G_{\mathrm{Q}}$-equivariant isomorphism

$$
H_{1}\left(\mathrm{Gr}_{I_{\ell}}^{>0} G_{\mathrm{Q}}, Q_{\ell}\right) \cong \bigoplus_{n \geqslant 1} H_{\mathrm{cts}}^{1}\left(G_{\mathrm{Q}}, \mathrm{Q}_{\ell}(2 n+1)\right)^{*} \otimes \mathrm{Q}_{\ell}(2 n+1) \cong \bigoplus_{n \geqslant 1} \mathrm{Q}_{\ell}(2 n+1) .
$$

The value of Soulé's element [33] of $H_{\mathrm{cts}}^{1}\left(G_{\mathrm{Q}}, \mathrm{Q}_{\ell}(2 n+1)\right)$ on the image of $s_{2 n+1}$ in $H_{\mathrm{cts}}^{1}\left(G_{\mathrm{Q}}, Q_{\ell}(2 n+1)\right)^{*} \otimes \mathrm{Q}_{\ell}(2 n+1)$ is nonzero.

In [16] Ihara constructs explicit elements $\sigma_{3}, \sigma_{5}, \sigma_{7}, \ldots$ of $\mathrm{Gr}_{I_{\ell}}^{>0} G_{\mathrm{Q}}$, which are sometimes called Soule elements. He studies the nonvanishing of the Lie brackets of these elements and asks [16, p. 300] whether the $\sigma_{j}$ generate $\left(\mathrm{Gr}_{I_{\ell}}^{>0} G_{Q}\right) \otimes Q_{\ell}$ freely. In [17], he proves that if $\left(\mathrm{Gr}_{I_{\ell}}^{>0} G_{Q}\right) \otimes Q_{\ell}$ is free, then it is generated by the Soule elements. He also shows that the $\sigma_{j}$ generate an open subgroup of the image of the homomorphism $G_{\ell} \rightarrow \operatorname{Out}\left(\pi^{(\ell)} / L^{m+1} \pi^{(\ell)}\right)$ for each $m$, a consequence of Conjecture 1 . The techniques of this paper do not seem to shed light on the question of freeness.

Our basic tool, which we develop in Paragraphs 3, 3.1, and 4, is the theory of weighted completion of a profinite group $\Gamma$ with respect to a continuous, Zariski dense representation $\rho: \Gamma \rightarrow R\left(Q_{\ell}\right)$ into a $Q_{\ell}$-group endowed with a distinguished central cocharacter $w: \mathrm{G}_{m} \rightarrow R$. The weighted completion in this case consists of 
(i) a proalgebraic $Q_{\ell}$-group $\mathcal{G}$ which is an extension

$1 \rightarrow \mathcal{U} \rightarrow \mathcal{G} \rightarrow R \rightarrow 1$

of $R$ by a prounipotent group $\mathcal{U}$ with the property that $H_{1}(\mathcal{U})$, when viewed as a $\mathrm{G}_{m}$-module via $w$, has only negative weights in the sense of representation theory;

(ii) a continuous homomorphism $\tilde{\rho}: \Gamma \rightarrow \mathcal{G}\left(Q_{\ell}\right)$ which lifts $\rho$.

These data are required to be universal for homomorphisms of $\Gamma$ into such 'negatively weighted extensions' of $R$ by a prounipotent group. It is a variant of Deligne's notion of the relative Malcev completion, which is developed in [12, 13]. One can also view weighted completions of $\Gamma$ as the Tannakian fundamental group of an appropriate category of finite-dimensional $\Gamma$-modules over $Q_{\ell}$. This approach is explained in [14] - see also Remark 4.3.

Weighted completions arise naturally in Galois theory in many contexts, the simplest of which is the following:

(i) $\ell$ is a prime number,

(ii) $\Gamma=G_{\ell}$, the Galois group of the maximal algebraic extension of $Q$ unramified outside $\ell$,

(iii) $R=\mathrm{G}_{m}$,

(iv) $\rho: G_{\ell} \rightarrow \mathrm{G}_{m}\left(\mathrm{Q}_{\ell}\right)=\mathrm{Q}_{\ell}^{\times}$is the $\ell$-adic cyclotomic character,

(v) $w: \mathrm{G}_{m} \rightarrow \mathrm{G}_{m}$ is the homomorphism defined by $w(x)=x^{-2}$.

In Section 7 we show that the weighted completion is an extension

$$
1 \rightarrow \mathcal{K}_{\ell} \rightarrow \mathcal{A}_{\ell} \rightarrow \mathrm{G}_{m} \rightarrow 1,
$$

where the Lie algebra $\mathfrak{f}_{\ell}$ of $\mathcal{K}_{\ell}$ is isomorphic (noncanonically) to the completion of the Lie algebra generated by

$$
\bigoplus_{n \geqslant 0} H_{\mathrm{cts}}^{1}\left(G_{\ell}, Q_{\ell}(2 n+1)\right)^{*} \otimes Q_{\ell}(2 n+1) \cong \bigoplus_{n \geqslant 0} Q_{\ell}(2 n+1) .
$$

The most important property of weighted completions (or more generally, negatively weighted extensions) is that each module of such a group has a natural weight filtration, and that the weight graded functor is exact on the category of such modules. This exactness property, called strictness, is familiar from Deligne's mixed Hodge theory [4] as well as Galois theory. It is a key ingredient in the proof of Conjecture 1. The reason for taking the central cocharacter to be $x \rightarrow x^{-2}$ in the previous paragraph is to make the representation theoretic weights agree with the usual weights in algebraic geometry coming from Hodge theory and Galois theory.

The Lie algebra of a weighted completion is a module over the group via the adjoint action and therefore has a natural weight filtration. In particular, the Lie algebra $a_{\ell}$ of the weighted completion of $G_{\ell}$ has a weight filtration where $\mathfrak{f}_{\ell}=W_{-1} \mathfrak{a}_{\ell}$. The copy of $Q_{\ell}(2 n+1)$ in the previous paragraph lies in $W_{-2(2 n+1)} \mathfrak{f}_{\ell}$ 
and projects to a non-zero element in the $-2(2 n+1)$ th weight graded quotient of $\mathfrak{f}_{\ell}$. These together generate $\mathrm{Gr}_{\text {. }}^{W_{\ell}}$.

The first step in the proof of Conjecture 1 is to use the fact, due to Ihara [15], that the Galois representation (1) factors through $G_{\ell}$. This outer action induces one on the unipotent completion $\mathcal{P}$ over $Q_{\ell}$ of the geometric fundamental group of $\mathbb{P}^{1}-\{0,1, \infty\}$. One can define the filtration (2) of $G_{\ell}$ by considering this action. The representation of $G_{\ell}$ on the Lie algebra $\mathfrak{p}$ of $\mathcal{P}$ is negatively weighted in an appropriate sense and induces a homomorphism $\mathcal{A}_{\ell} \rightarrow$ Out $\mathcal{P}$ and a corresponding homomorphism $\mathfrak{a}_{\ell} \rightarrow$ OutDer $\mathfrak{p}$ on Lie algebras. Using strictness, one can show (see Section 8) that

$$
\left(\mathrm{Gr}_{I_{\ell}}^{m} G_{\ell}\right) \otimes_{Z_{\ell}} Q_{\ell} \cong \operatorname{Gr}_{-2 m}^{W} \operatorname{im}\left\{\mathfrak{f}_{\ell} \rightarrow \text { OutDer } \mathfrak{p}\right\} \cong \operatorname{im}\left\{\mathrm{Gr}_{-2 m}^{W} \mathfrak{f}_{\ell} \rightarrow \text { OutDer Gr}{ }_{\bullet}^{W} \mathfrak{p}\right\}
$$

whenever $m>0$. Conjecture 1 then follows from the computation of $\mathrm{Gr}_{\bullet}^{W} \mathfrak{a}_{\ell}$ in Section 7.

Goncharov's Conjecture is similar to Ihara's and concerns the action (as distinct from the outer action) of the Galois group of the kernel of the $\ell$-adic cyclotomic character, $Q\left(\mu_{\ell}\right)$, on the $\ell$-adic prounipotent completion $\mathcal{P}$ of $\pi_{1}\left(\mathbb{P}^{1}(\mathrm{C})-\right.$ $\{0,1, \infty\}, \overrightarrow{01})$ with base point the tangent vector at 0 pointing towards 1 . In addition to conjecturing that the Lie algebra of the Zariski closure of the image of $G_{Q}\left(\mu_{e}\right)$ in Aut $\mathcal{P}$ is generated by elements $s_{3}, s_{5}, s_{7}, \ldots$, he conjectures that it is freely generated by them. We are able to prove the generation part of his conjecture, but not the freeness. Goncharov's Conjecture is considered in Paragraph 8.5 .

In Section 9 we prove $\ell$-adic versions of two related conjectures of Deligne, $[5,8.2$, p. 163] and [5, 8.9.5, p. 168], concerning mixed Tate motives over the spectrum of the ring of $S$-integers of a number field. As was later pointed out to us by Goncharov, these had already been proved by Beilinson and Deligne in their unpublished manuscript [1]. Our approach via weighted completion is different, but equivalent, to the approach of Beilinson and Deligne. Notably one should mention that Goncharov [10] has used the work of Voevodsky [37] and Levine [20] to construct the category of mixed Tate motives over the spectrum of a number field. It suggests a working definition of the category of mixed Tate motives over the spectrum of a ring of $S$-integers in a number field.

Our proof of Conjecture 1 can be conceptualized by saying that the unipotent completion of the fundamental group of $\pi_{1}\left(\mathbb{P}^{1}-\{0,1, \infty\}, \overrightarrow{01}\right)$ should be a mixed Tate motive over Spec Z, and therefore a module over the Tannakian fundamental group of the category of mixed Tate motives over Spec Z. As was pointed out to us by a referee, once one knows that the unipotent completion of the fundamental group of $\pi_{1}\left(\mathbb{P}^{1}-\{0,1, \infty\}, \overrightarrow{01}\right)$ is a mixed Tate motive over Spec Z in the sense of Goncharov, Conjecture 1 will follow by some arguments including the strictness of weight filtrations. Our approach here is more direct, treating only $\ell$-adic Galois representations. 
Deligne's Conjectures concern the category of mixed Tate motives over the spectrum $X_{F, S}:=\operatorname{Spec} \mathcal{O}_{F}-S$ of the ring of $S$-integers of a number field $F$. His conjectures essentially say that

(i) there should be a category of mixed Tate motives over $X_{F, S}$;

(ii) the tannakian fundamental group of this category should be a proalgebraic Q-group which is an extension of $\mathrm{G}_{m}$ by a prounipotent group;

(iii) the Lie algebra of this prounipotent radical should be free and its first cohomology is isomorphic to $\bigoplus_{n>0} K_{n}\left(X_{F, S}\right) \otimes \mathrm{Q}(-n)$.

Let $X_{F, S}[1 / \ell]$ denote $X_{F, S}$ minus all primes over $\ell$. In Section 9, we define the category of $\ell$-adic mixed Tate modules over $X_{F, S}$ to be the category whose objects are $\pi_{1}\left(X_{F, S}[1 / \ell]\right)$-modules $M$ over $Q_{\ell}$ endowed with a weight filtration $W$. by $\pi_{1}\left(X_{F, S}[1 / \ell]\right)$-submodules such that all odd weight graded quotients of $M$ vanish and the $(-2 m)$ th graded quotient is a sum of a finite number copies of $Q_{\ell}(m)$. In addition, we require that all representations be crystalline at all primes $\mathfrak{p}$ outside $S$ that lie over $\ell$.

When $S$ contains all primes that lie over $\ell$, the Tannakian fundamental group of the category of $\ell$-adic mixed Tate modules is the weighted completion $\mathcal{A}_{F, S}$ of $\pi_{1}\left(X_{F, S}\right)$ with respect to the cyclotomic character. This is shown, in Section 7, to be an extension of $\mathrm{G}_{m}$ by a prounipotent $Q_{\ell}$-group whose Lie algebra is isomorphic to a completion of the free Lie algebra generated by

$$
\bigoplus_{n>0} H_{\mathrm{et}}^{1}\left(X_{F, S}, \mathrm{Q}_{\ell}(n)\right)^{*} \otimes \mathrm{Q}_{\ell}(n) .
$$

This group is related to the algebraic $K$-theory of $X_{F, S}$ by the regulators

$$
c_{1}: K_{2 n-1}\left(X_{F, S}\right) \rightarrow H_{\mathrm{et}}^{1}\left(X_{F, S}, \mathrm{Q}_{\ell}(n)\right) .
$$

Soulé's result [33] when $\ell$ is odd, and results of Rognes and Weibel [27] when $\ell=2$, imply that these induce isomorphisms

$$
\bigoplus_{n>0} K_{2 n-1}\left(X_{F, S}\right) \otimes Q_{\ell} \stackrel{\simeq}{\longrightarrow} \bigoplus_{n>0} H_{\mathrm{et}}^{1}\left(X_{F, S}, \mathrm{Q}_{\ell}(n)\right) .
$$

This yields a proof of an $\ell$-adic version of Deligne's conjectures similar to the unpublished proof of Beilinson and Deligne [1].

There is an appendix on unipotent completion where we collect and prove results we need. Many of these must be well known but we know of no good references, while other results may be new. One innovation is that we introduce the notion of the $\ell$-adic unipotent completion of a profinite group and prove a comparison theorem which states that if $\Gamma$ is a finitely generated group, then the $\ell$-adic unipotent completion of the profinite (or pro- $\ell$ ) completion of $\Gamma$ is isomorphic to the $Q_{\ell}$-form of the ordinary unipotent completion of $\Gamma$.

There is a second appendix in which we prove some results about the continuous cohomology of the Galois groups $G_{F, S}$ which are surely well known to the experts, but for which we could not find suitable references. 
Conventions. Unless mentioned otherwise, all fields in this paper will be of characteristic zero.

The functor $H_{1}$ when applied to a group, Lie algebra, etc., will denote the maximal abelian quotient. In the topological case, it will denote the quotient by the closure of the commutator subgroup or subalgebra, as appropriate.

Following the conventions in Hodge theory (cf. [4]), we shall denote increasing filtrations with a subscript index:

$$
\text { W. } M: \cdots \subseteq W_{m-1} M \subseteq W_{m} M \subseteq \cdots
$$

and decreasing filtrations with a superscript index:

$$
I^{\bullet} M: \cdots \supseteq I^{m} M \supseteq I^{m+1} M \supseteq \cdots
$$

Their associated gradeds are defined by

$$
\mathrm{Gr}_{\bullet}^{W} M:=\bigoplus_{m \in \mathbb{Z}} W_{m} M / W_{m-1} M \text { and } \operatorname{Gr}_{I}^{\bullet} M:=\bigoplus_{m \in \mathbb{Z}} I^{m} M / I^{m+1} M .
$$

Throughout this paper, $k$ denotes a field of characteristic zero.

\section{Preliminaries on Proalgebraic Groups}

In this paper, a group scheme or equivalently a $k$-group scheme means an affine group scheme over $k$. An algebraic group over a field $k$ or an algebraic $k$-group means an affine group scheme of finite type over $k$. A proalgebraic group is a projective limit of algebraic groups over $k$. It is known that the category of proalgebraic groups over $k$ is equivalent to the category of affine group schemes over $k$, and any proalgebraic group is a projective limit of a projective system consisting of surjective morphisms (cf. [6, Corollary 2.7, p. 128]).

If $\mathcal{G}=\overleftarrow{\alpha}_{\alpha}^{\lim } G_{\alpha}$, then the set of its $k$-rational points satisfies

$$
\mathcal{G}(k)=\underbrace{\lim }_{\alpha} G_{\alpha}(k) .
$$

The Zariski closure of a subgroup $S$ of $\mathcal{G}(K), K$ an extension field of $k$, is the smallest proalgebraic $k$-group that contains it - it is the projective $\operatorname{limit} \lim S_{\alpha}$ where $S_{\alpha}$ is the $k$-Zariski closure of the image of $S$ in $G_{\alpha}(K)$.

The Lie algebra $g$ of the proalgebraic $k$-group

$$
\mathcal{G}=\varlimsup_{\alpha}^{\lim } G_{\alpha},
$$

where each $G_{\alpha}$ is algebraic, is simply the inverse limit

$$
\underset{\alpha}{\lim } g_{\alpha}
$$

of the Lie algebras of the $G_{\alpha}$. It will be regarded as a topological Lie algebra with the topology defined by the inverse limit, where each $\mathfrak{g}_{\alpha}$ has the discrete topology. 
If each $G_{\alpha}$ is unipotent, we say that $\mathcal{G}$ is prounipotent. The Lie algebra of a prounipotent group is the inverse limit of nilpotent Lie algebras (in this paper a nilpotent Lie algebra means a finite-dimensional nilpotent Lie algebra). Such a Lie algebra is said to be pronilpotent.

The well known correspondence between unipotent groups of $k$ and nilpotent Lie algebras over $k$, which assigns to a unipotent group its Lie algebra, extends easily to a correspondence between prounipotent groups over $k$ and pronilpotent Lie algebras over $k$ (cf. [26, Appendix A]).

A graded Lie algebra is a Lie algebra in the category of graded vector spaces. It is of finite type if each of its graded quotients is finite-dimensional. The following results are needed in the sequel. The first is an immediate consequence of the elementary fact that a homomorphism $\mathfrak{n}_{1} \rightarrow \mathfrak{n}_{2}$ between finite-dimensional nilpotent Lie algebras is surjective if and only if the induced map $H_{1}\left(\mathfrak{n}_{1}\right) \rightarrow H_{1}\left(\mathfrak{n}_{2}\right)$ is surjective.

PROPOSITION 2.1. If $\mathfrak{n}=\bigoplus_{m>0} \mathfrak{n}_{m}$ is a graded Lie algebra, then the image of any section of the canonical surjection $\mathfrak{n} \rightarrow H_{1}(\mathfrak{n})$ generates $\mathfrak{n}$.

Any such section induces a homomorphism from the free Lie algebra generated by $H_{1}(\mathfrak{n})$. Thus we have:

COROLLARY 2.2. If $\mathfrak{n}=\bigoplus_{m>0} \mathfrak{n}_{m}$ is a graded Lie algebra, then there is a free

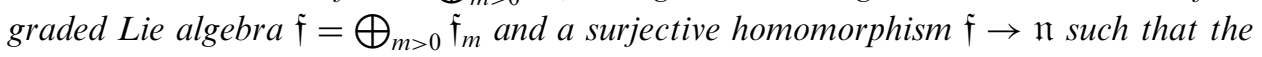
induced map $H_{1}(\mathfrak{f}) \rightarrow H_{1}(\mathfrak{n})$ is an isomorphism.

Finally, we recall the Levi decomposition [2, p. 158].

PROPOSITION 2.3. Every algebraic k-group $G$ is an extension of a reductive $k$-group $R$ (called the Levi quotient) by a unipotent group $U$ (called the unipotent radical),

$$
1 \rightarrow U \rightarrow G \rightarrow R \rightarrow 1 .
$$

This extension is split and any two splittings differ by conjugation by an element of $U(k)$.

\section{Negatively Weighted Extensions and Weight Filtrations}

Let $R$ be an algebraic $k$-group. Denote $\mathbb{G}_{m / k}$ by $\mathrm{G}_{m}$. Suppose that $w: \mathrm{G}_{m} \rightarrow R$ is a central cocharacter - that is, a homomorphism whose image is contained in the center of $R$.

We shall denote by $Q^{m}$ the one-dimensional irreducible representation of $\mathrm{G}_{m}$ on which it acts by the $m$ th power of the standard character. We shall denote the $Q^{m}$ isotypical component of a $\mathrm{G}_{m}$-module $V$ by $V_{m}$.

Suppose that

$$
1 \rightarrow U \rightarrow G \rightarrow R \rightarrow 1
$$


is an extension of $R$ by a unipotent group in the category of algebraic $k$-groups. The first homology of $U$ is an $R$-module, and therefore a $\mathrm{G}_{m}$-module via the homomorphism $w$. Thus we can write

$$
H_{1}(U)=\bigoplus_{m \in Z} H_{1}(U)_{m} .
$$

We shall say that this extension is negatively weighted with respect to $w$ if $H_{1}(U)_{m}$ vanishes whenever $m \geqslant 0$.

We shall say that a proalgebraic group which is an extension of $R$ by a prounipotent group $\mathcal{U}$ is negatively weighted if it is an inverse limit of negatively weighted algebraic groups.

If $w$ is trivial, then there are no nontrivial negatively weighted extensions of $R$.

\subsection{THE WEIGHT FILTRATION}

Suppose that an algebraic $k$-group $G$ is a negatively weighted extension of $R$ with respect to the central cocharacter $w: \mathrm{G}_{m} \rightarrow R$. In this section, we show that representations of a negatively weighted extension of $R$ have a natural weight filtration, and that morphisms between such modules are strict with respect to this weight filtration.

LEMMA 3.1. There is a lift of the homomorphism $w: \mathrm{G}_{m} \rightarrow R$ to a homomorphism $\tilde{w}: \mathrm{G}_{m} \rightarrow G$, and any two such liftings are conjugate by an element of $U(k)$.

Proof. This can be seen by pulling back the extension $U \rightarrow G \rightarrow R$ along $w$ and then applying Proposition 2.3.

Fix such a splitting $\tilde{w}$. Each finite-dimensional $G$-module $V$ can then be regarded as a $\mathrm{G}_{m}$-module via $\tilde{w}$ and can therefore be decomposed

$$
V=\bigoplus_{n \in Z} V_{n}
$$

under the $\mathrm{G}_{m}$-action. Define the weight filtration of $V$ by

$$
W_{n} V=\bigoplus_{m \leqslant n} V_{m} .
$$

A priori, this filtration depends on $\tilde{w}$ but we will show, in Proposition 3.8, that it does not.

The $n$th weight graded quotient of $V$ is defined by

$$
\mathrm{Gr}_{n}^{W} V:=W_{n} V / W_{n-1} V .
$$

The inclusion $V_{n} \hookrightarrow V$ induces a natural isomorphism

$$
\mathrm{Gr}_{n}^{W} V \cong V_{n} .
$$

It follows directly from the representation theory of $\mathrm{G}_{m}$ that, for a fixed splitting $\tilde{w}$, the weight splitting (3) is compatible with Hom and tensor products. 
PROPOSITION 3.2. If $U$ and $V$ are $G$-modules, then

and

$$
\operatorname{Hom}(U, V)_{n}=\bigoplus_{r-m=n} \operatorname{Hom}\left(U_{m}, V_{r}\right)
$$

$$
(U \otimes V)_{n}=\bigoplus_{r+m=n} U_{m} \otimes V_{r} .
$$

Moreover, if $f: V \rightarrow W$ is G-equivariant, then $f\left(V_{n}\right) \subset W_{n}$ for all $n$.

An immediate consequence is that the weight filtration is compatible with respect to Hom and tensor products.

COROLLARY 3.3. If $U$ and $V$ are $G$-modules, then

$$
W_{n} \operatorname{Hom}(U, V)=\left\{\phi \in \operatorname{Hom}(U, V): \phi\left(W_{r} U\right) \subseteq W_{r+n} V \text { for all } r \in \mathbb{Z}\right\}
$$

and

$$
W_{n}(U \otimes V)=\sum_{r+s=n} W_{r} U \otimes W_{s} V .
$$

We can apply this to the adjoint action to decompose $\mathfrak{g}$, the Lie algebra of $G$, and $\mathfrak{u}$, the Lie algebra of the negative unipotent part $U$ :

$$
\mathfrak{g}=\bigoplus_{n \in \mathbb{Z}} \mathfrak{g}_{n} \quad \text { and } \quad \mathfrak{u}=\bigoplus_{n \in \mathbb{Z}} \mathfrak{u}_{n}
$$

Denote the Lie algebra of $R$ by $r$.

PROPOSITION 3.4. With notation as above,

(i) if $V$ is a G-module, $x \in \mathfrak{g}_{n}$ and $v \in V_{m}$, then $x \cdot v \in V_{n+m}$;

(ii) $\mathfrak{g}$ is a graded Lie algebra; that is, if $x \in \mathfrak{g}_{n}$ and $y \in \mathfrak{g}_{m}$, then $[x, y] \in \mathfrak{g}_{n+m}$;

(iii) $\mathfrak{u}$ is a sub graded Lie algebra of $\mathfrak{g}$, that is, $\mathfrak{u}_{m} \subseteq \mathfrak{g}_{m}$;

(iv) if $n \geqslant 0$, then $\mathfrak{u}_{n}=0$;

(v) the graded quotients of $\mathfrak{g}$ are given by

$$
\mathfrak{g}_{n}=\left\{\begin{array}{cc}
0, & n>0 ; \\
\mathfrak{r}, & n=0 ; \\
\mathfrak{u}_{n}, & n<0 .
\end{array}\right.
$$

Proof. The first statement follows from the previous proposition as the mapping $\mathfrak{g} \otimes V \rightarrow V$ is $G$-equivariant. The second follows from the first by taking $V$ to be the adjoint representation. The third statement is clear.

Set

$$
\mathfrak{u}_{<0}=\bigoplus_{n<0} \mathfrak{u}_{n}
$$


The inclusion of this into $\mathfrak{u}$ induces a surjection on $H_{1}$ as $H_{1}(\mathfrak{u})$ has all negative weights. Since $\mathfrak{u}$ and $\mathfrak{t}_{<0}$ are both nilpotent, this implies that they are equal. This proves the fourth statement.

The exact sequence $0 \rightarrow \mathfrak{t} \rightarrow \mathfrak{g} \rightarrow \mathfrak{r} \rightarrow 0$ is an exact sequence of graded $\mathfrak{g}$ modules. Since $w$ is central in $R$, it follows that $\mathfrak{r}=\mathfrak{r}_{0}$. Since $\mathfrak{u}$ has only negative weights, it follows that the projection $\mathfrak{g} \rightarrow \mathfrak{r}$ induces an isomorphism $\mathfrak{g}_{0} \cong \mathfrak{r}$ and that $\mathfrak{g}_{n}=\mathfrak{u}_{n}$ whenever $n \neq 0$.

COROLLARY 3.5. With notation as above, $W_{\bullet} \mathrm{g}$ is a filtration of $\mathrm{g}$ by Lie ideals, and we have $\mathfrak{g}=W_{0} \mathfrak{g}, \mathfrak{u}=W_{-1} \mathfrak{g}$, and $\mathfrak{r} \cong \mathrm{Gr}_{0}^{W} \mathfrak{g}$.

LEMMA 3.6. For every choice of lift $\tilde{w}$, each term $W_{n} V$ of the weight filtration of the G-module $V$ is a g-module, and $U$ acts trivially on its associated graded $\mathrm{Gr}^{W} V$.

Proof. Since $\mathfrak{i t}=W_{-1} \mathfrak{g}$ and since the action $\mathfrak{g} \otimes V \rightarrow V$ is compatible with the gradings, each term $W_{n} V$ of the weight filtration is a $\mathfrak{g}$-module. Since $\mathfrak{u}=W_{-1} \mathfrak{g}$, the image of the action $\mathfrak{u} \otimes W_{n} V \rightarrow W_{n} V$ is contained in $W_{n-1} V$, from which the result follows.

The Levi decomposition extends to negatively weighted extensions.

LEMMA 3.7. If $1 \rightarrow U \rightarrow G \rightarrow R \rightarrow 1$ is a negatively weighted extension with respect to the central cocharacter $w: \mathrm{G}_{m} \rightarrow R$, then the projection $G \rightarrow R$ splits, and any two splittings are conjugate by an element of $U$.

Proof. If $R$ is reductive, this follows directly from the classical Levi decomposition (Prop. 2.3). When $R$ connected, the existence of the splitting follows from the existence of a splitting of $\mathfrak{g} \rightarrow \mathfrak{r}$ as $U$ is simply connected. The existence of such a Lie algebra splitting follows by first taking a lifting $\tilde{w}$ : $\mathrm{G}_{m} \rightarrow G$ of $w$, and then decomposing $\mathfrak{g}$ under the restriction of the adjoint action to $\mathrm{G}_{m}$ via $\tilde{w}$. The summand of weight zero (the invariant part) is a Lie algebra (as the bracket preserves weights) and projects isomorphically to $r$.

First we will prove that any two liftings have to be conjugate by an element of $U$. The intersection of $U$ with the centralizer $C(\tilde{w})$ of a lift $\tilde{w}: \mathrm{G}_{m} \rightarrow G$ of $w$ is just the identity as the Lie algebra of $U$ has strictly negative weights with respect to $\tilde{w}$ under the adjoint action. It follows that if $s: R \rightarrow G$ is a splitting and $\tilde{w}=s \circ w$, then $s(R)=C(\tilde{w})$. Since the lifts $\tilde{w}$ are unique up to conjugation by an element of $U$, any two splitting of $G \rightarrow R$ have to be conjugate by an element of $U$.

To prove the existence of the splitting in general, first take a Levi decomposition

$$
R \cong \bar{R} \times W
$$

of $R$, where $\bar{R}$ is reductive and $W$ unipotent. This also induces a Levi decomposition

$$
R^{o} \cong \bar{R}^{o} \ltimes W
$$


of $R^{o}$, the connected component of the identity. By the connected case, we can lift $R^{o}$ to $G$. In particular, we can lift $W$ to $G$. Since $W$ and $U$ are unipotent, $\bar{R}$ is the reductive quotient of $G$. By the classical Levi decomposition, we have a splitting of $G \rightarrow \bar{R}$. The subtlety is that we have to choose this splitting so that its image normalizes the lift of $W$.

The splittings $\bar{R} \rightarrow G$ and $R^{o} \rightarrow G$ determine lifts $\tilde{w}_{0}$ and $\tilde{w}_{1}$ of $w$. By conjugating one of the splittings by an element of $U$, we may assume they are the same. But then the images of both splittings lie in the centralizer $C\left(\tilde{w}_{0}\right)$. It follows that the image projection $C\left(\tilde{w}_{0}\right) \rightarrow R$ is surjective, and therefore an isomorphism. Inverting this isomorphism provides the splitting.

PROPOSITION 3.8. The weight filtration of a G-module $V$ is independent of the choice of the lift $\tilde{w}$ of $w$ and has the property that each $W_{m} V$ is a G-module. Moreover, each $\mathrm{Gr}_{m}^{W} V$ is an R-module of weight $m$.

Proof. Suppose that $\tilde{w}^{\prime}: \mathrm{G}_{m} \rightarrow G$ is another lift of $w$. Then, by Lemma 3.1, there is $u \in U(k)$ such that $\tilde{w}^{\prime}=u \tilde{w} u^{-1}$. Write

$$
V=\bigoplus_{n \in \mathbb{Z}} V_{n}^{\prime}
$$

where $V_{n}^{\prime}$ is the subspace of $V$ where $\mathrm{G}_{m}$ acts with weight $n$ via $\tilde{w}^{\prime}$. Then $V_{n}^{\prime}=u V_{n}$. Since each term of the weight filtrations associated to $w$ and $\tilde{w}$ are $G$-modules, it follows that, for each $n$,

$$
\bigoplus_{m \leqslant n} V_{m}^{\prime}=\bigoplus_{m \leqslant n} u V_{m}=u\left(\bigoplus_{m \leqslant n} V_{m}\right) \subseteq \bigoplus_{m \leqslant n} V_{m} .
$$

The reverse inclusion follows by reversing the roles of $w$ and $\tilde{w}$. The equality of the weight filtrations follows.

To prove the second assertion, we choose a splitting $s: R \rightarrow G$ of the canonical projection $G \rightarrow R$, whose existence is assured by Lemma 3.7. Choose the lift $\tilde{w}$ to be the composite of $w$ : $\mathrm{G}_{m} \rightarrow R$ with the splitting $s$. Let $V=\bigoplus V_{n}$ be the corresponding weight decomposition of $V$. Since $w$ is central in $R$, it follows that the image of $s$ preserves this decomposition and that each $W_{m} V$ is an (im $\left.s\right)$-module. The result follows from Lemma 3.6.

The last assertion is clear.

\subsection{THE PROALGEBRAIC CASE}

Suppose now that $\mathcal{G}$ is a proalgebraic $k$-group which is a negatively weighted extension of the algebraic $k$-group $R$ and that $V$ is a finite-dimensional $\mathcal{G}$-module. Note that $\mathcal{G}$ is the projective limit of algebraic $k$-groups $G_{\alpha}$, each of which is a (necessarily negatively weighted) extension of $R$. Since $V$ is finite-dimensional, it is a $G_{\alpha}$-module 
for some $\alpha$, and therefore has a weight filtration. It is easily seen that this weight filtration is independent of $\alpha$. We therefore have:

THEOREM 3.9. If $\mathcal{G}$ is a proalgebraic k-group which is a negatively weighted extension of the algebraic $k$-group $R$, then every finite-dimensional $\mathcal{G}$-module has a natural weight filtration $W$. by $\mathcal{G}$-submodules. This has the following properties:

(i) the action of $\mathcal{G}$ on $\mathrm{Gr}_{.}^{W} V$ factors through $\mathcal{G} \rightarrow R$;

(ii) the action of $\mathrm{G}_{m}$ on $\mathrm{Gr}_{m}^{W} V$ via $w$ is of weight $m$;

(iii) the weight filtration is compatible with $\mathrm{Hom}$ and $\otimes$ in that it obeys the rules in Corollary 3.3.

The weight filtration extends naturally to two different classes of infinite-dimensional representations of $\mathcal{G}-$ viz, those representations that are projective or inductive limits of finite-dimensional $\mathcal{G}$-modules. In particular, the Lie algebras $\mathfrak{g}$ of $\mathcal{G}$ and ut of $\mathcal{U}$ have natural weight filtrations.

\subsection{MORPHISMS}

The weight filtration has nice naturality and exactness properties. The following setting is a little technical, but convenient for our applications.

Suppose that $R_{1}$ and $R_{2}$ are algebraic $k$-groups with distinguished central cocharacters $w_{i}: \mathrm{G}_{m} \rightarrow R_{i}$ and that $\mathcal{G}_{i}$ is a proalgebraic $k$-group where $i=1,2$. Suppose that $\mathcal{G}_{i} \rightarrow R_{i}$ is an extension (not necessarily negatively weighted) of $R_{i}$, where $i=1$, 2. Suppose that $\Phi: \mathcal{G}_{1} \rightarrow \mathcal{G}_{2}$ and $\phi: R_{1} \rightarrow R_{2}$ are homomorphisms such that the diagramz

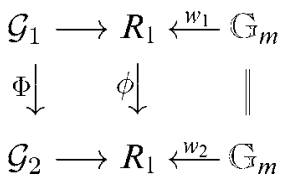

commutes.

The following result says that, in some sense, negatively weighted extensions are closed under subs and quotients. It is easily proved.

LEMMA 3.10. Suppose that $\Phi: \mathcal{G}_{1} \rightarrow \mathcal{G}_{2}$ is as above.

(i) If $\Phi$ is an inclusion and $\mathcal{G}_{2}$ is a negatively weighted extension of $R_{2}$ with respect to $w_{2}$, then $\mathcal{G}_{1}$ is a negatively weighted extension of $R_{1}$ with respect to $w_{1}$;

(ii) If $\Phi$ is a surjection and $\mathcal{G}_{1}$ is a negatively weighted extension of $R_{1}$ with respect to $w_{1}$, then $\mathcal{G}_{2}$ is a negatively weighted extension of $R_{2}$ with respect to $w_{2}$. 
A $\Phi$-homomorphism from a $\mathcal{G}_{1}$-module $V_{1}$ to a $\mathcal{G}_{2}$-module $V_{2}$ is a $\mathcal{G}_{1}$ homomorphism, where $V_{2}$ is regarded as a $\mathcal{G}_{1}$-module via $\Phi$.

We can form a category: The objects are 4-tuples $(V, R, w, \mathcal{G})$ where $R$ is an algebraic $k$-group, $w: \mathrm{G}_{m} \rightarrow R$ a central cocharacter, $\mathcal{G}$ a proalgebraic group which is a negative extension of $R$, and where $V$ is a finite-dimensional $\mathcal{G}$-module. Morphisms between such modules are the $\Phi$ homomorphisms defined above. We shall call this the category of weighted modules. Note that every object of this category has a natural weight filtration.

\subsection{STRICTNESS}

Strictness is a key ingredient in our proof of Conjecture 1. A linear mapping $f:\left(V_{1}, W_{\bullet}\right) \rightarrow\left(V_{2}, W_{\bullet}\right)$ between two filtered vector spaces is said to be strict with respect to the filtrations $W_{\text {. }}$ if it is filtration preserving and if

$$
\operatorname{im} f \cap W_{m} V_{2}=f\left(W_{m} V_{1}\right)
$$

for all $m \in Z$.

A filtration $W$. of a vector space induces a filtration on each subspace $A \hookrightarrow V$ and each quotient $q: V \rightarrow B$ by

$$
W_{m} A:=A \cap W_{m} V \quad \text { and } \quad W_{m} B=q\left(W_{m} V\right) .
$$

PROPOSITION 3.11. If $f:\left(V_{1}, W_{\bullet}\right) \rightarrow\left(V_{2}, W_{\bullet}\right)$ is strict with respect to $W_{\bullet}$, then there are natural isomorphisms

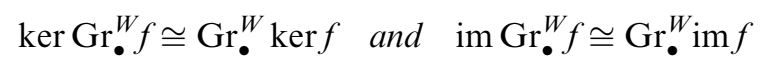

of graded vector spaces.

THEOREM 3.12. Each homomorphism $f: V_{1} \rightarrow V_{2}$ in the category of weighted modules preserves the weight filtration and is strict with respect to it. Consequently, the functors

$$
V \mapsto \operatorname{Gr}_{m}^{W} V, \quad V \mapsto W_{m} V, \quad \text { and } \quad V \mapsto V / W_{m} V
$$

are all exact on the category of finite-dimensional $\mathcal{G}$-modules.

Proof. We use the notation of Paragraph 3.3. Since $V_{1}$ and $V_{2}$ are finite-dimensional, we may assume that $\mathcal{G}_{1}$ and $\mathcal{G}_{2}$ are algebraic. To prove that the weight filtration is preserved by the morphism $f: V_{1} \rightarrow V_{2}$, choose a lift $\tilde{w}_{1}: \mathrm{G}_{m} \rightarrow G_{1}$, and define $\tilde{w}_{2}$ to be $\Phi \circ \tilde{w}_{1}$. Then both $V_{1}$ and $V_{2}$ become $\mathbb{G}_{m}$-modules, and $f$ is $\mathrm{G}_{m}$-equivariant. The first assertion follows. Strictness follows similarly as $f$ becomes a map of graded vector spaces after choosing the lift $\tilde{w}$.

The exactness of $\mathrm{Gr}_{n}^{W}$ follows for similar reasons. If

$$
0 \rightarrow V^{\prime} \rightarrow V \rightarrow V^{\prime \prime} \rightarrow 0
$$


is an exact sequence of weighted modules, one can choose compatible liftings of the central cocharacters, so that the exact sequence becomes an exact sequence of $\mathrm{G}_{m}$-modules. The sequence

$$
0 \rightarrow V_{n}^{\prime} \rightarrow V_{n} \rightarrow V_{n}^{\prime \prime} \rightarrow 0
$$

is thus exact for all $n$. The exactness of $\mathrm{Gr}_{n}^{W}$ follows as the $n$th weight graded quotient of a module is naturally isomorphic to its weight $n$ part.

This theorem extends to certain infinite-dimensional representations of such groups. There are two appropriate categories of such $\mathcal{G}$-modules. Namely, the category of projective limits of finite-dimensional representations of $\mathcal{G}$, and the category of inductive limits of finite-dimensional representations of $\mathcal{G}$. In both cases, there is a weight filtration and morphisms are strict with respect to it.

\section{Weighted Completion}

We will work only in the profinite setting as that is where our examples lie, although there is an obvious analogue in the discrete case. Denote $\mathrm{G}_{m / Q_{\ell}}$ by $\mathrm{G}_{m}$. Suppose that:

(i) $\Gamma$ is a profinite group;

(ii) $R$ is an algebraic group defined over $Q_{\ell}$;

(iii) $w: \mathrm{G}_{m} \rightarrow R$ is a central cocharacter;

(iv) $\rho: \Gamma \rightarrow R\left(Q_{\ell}\right)$ is a continuous homomorphism with Zariski dense image.

DEFINITION 4.1. The weighted completion of $\Gamma$ with respect to $\rho$ and $w$ is a proalgebraic $\mathrm{Q}_{\ell}$-group $\mathcal{G}$, which is an extension of $R$ by a prounipotent group $\mathcal{U}$, and a continuous homomorphism $\tilde{\rho}: \Gamma \rightarrow \mathcal{G}\left(Q_{\ell}\right)$ which lifts $\rho$ :

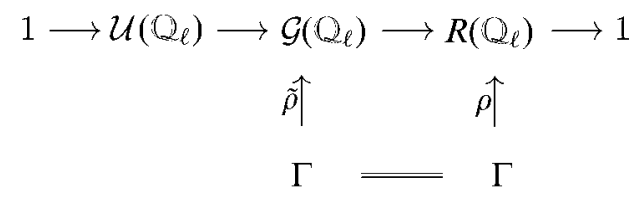

It is characterized by its universal mapping property: if $G$ is a proalgebraic $Q_{\ell}$-group which is a negatively weighted extension of $R$ (with respect to $w$ ) by a prounipotent group, and if $\phi: \Gamma \rightarrow G\left(Q_{\ell}\right)$ is a homomorphism that lifts $\rho$, then there is a unique homomorphism of proalgebraic groups $\Phi: \mathcal{G} \rightarrow G$ that commutes with the projections to $R$ and such that $\phi=\Phi \tilde{\rho}$.

DEFINITION 4.2. A $Q_{\ell} \Gamma$-module $V$ is a $Q_{\ell}$-vector space with a continuous $\Gamma$-action. A weighted $\Gamma$-module is a finite-dimensional $Q_{\ell} \Gamma$-modules $V$ with an 
increasing filtration $W \cdot$ by $\Gamma$-submodules where, for each $n$, the action of $\Gamma$ on $\mathrm{Gr}_{n}^{W} V$ factors through an action of $R$ via $\rho$ and where

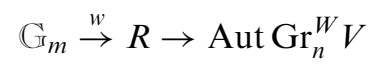

is the $n$th power of the standard character.

Morphisms between weighted $\Gamma$-modules are those as $\Gamma$-modules.

Remark 4.3. The weighted completion of $\Gamma$ with respect to $\rho: \Gamma \rightarrow R\left(\mathrm{Q}_{\ell}\right)$ can also be defined to be the Tannakian fundamental group of the category of weighted $\Gamma$-modules. This is proved in [14].

PROPOSITION 4.4. The weighted completion of $\Gamma$ with respect to $\rho: \Gamma \rightarrow R\left(Q_{\ell}\right)$ and w: $\mathrm{G}_{m} \rightarrow R$ always exists.

Proof. Consider the category whose objects are pairs

$$
\left(G \rightarrow R, \phi: \Gamma \rightarrow G\left(Q_{\ell}\right)\right),
$$

where $G$ is an algebraic $Q_{\ell}$-group which is a negatively weighted extension of $R$ and where $\phi$ is a continuous, Zariski dense representation which lifts $\rho: \Gamma \rightarrow R\left(Q_{\ell}\right)$. A morphism

$$
F:\left(G_{1} \rightarrow R, \phi_{1}: \Gamma \rightarrow G_{1}\left(Q_{\ell}\right)\right) \rightarrow\left(G_{2} \rightarrow R, \phi_{2}: \Gamma \rightarrow G_{2}\left(Q_{\ell}\right)\right)
$$

consists of a homomorphism $f: G_{1} \rightarrow G_{2}$ of $Q_{\ell}$-groups that is compatible with the projections to $R$ and satisfies $\phi_{2}=f \cdot \phi_{1}$.

Since the image of $\phi$ is Zariski dense, there is at most one morphism between any two objects. In addition, there is fibered product:

$$
\left(G_{1} \rightarrow R, \phi_{1}: \Gamma \rightarrow G_{1}\left(Q_{\ell}\right)\right) \times_{R}\left(G_{2} \rightarrow R, \phi_{2}: \Gamma \rightarrow G_{2}\left(Q_{\ell}\right)\right)
$$

is defined to be

$$
\left(G \rightarrow R, \phi: \Gamma \rightarrow G\left(Q_{\ell}\right)\right),
$$

where $G$ is the Zariski closure of the image of

$$
\phi_{1} \times_{R} \phi_{2}: \Gamma \rightarrow\left(G_{1} \times_{R} G_{2}\right)\left(Q_{\ell}\right) .
$$

It follows that this category is a projective system. The weighted completion of $\Gamma$ with respect to $\rho$ is then $\left(\mathcal{G}, \hat{\rho}: \Gamma \rightarrow \mathcal{G}\left(Q_{\ell}\right)\right)$, the projective limit of all objects of this category.

\subsection{COHOMOLOGY OF g AND $u$}

We shall view $\mathfrak{g}$ and $\mathfrak{u}$ as topological Lie algebras where neighbourhood of zero are the kernels $\mathfrak{n}$ of the canonical homomorphisms to the finite-dimensional quotients of 
$g$ that are extensions

$0 \rightarrow \mathfrak{u} / \mathfrak{n} \rightarrow \mathfrak{g} / \mathfrak{n} \rightarrow \mathfrak{r} \rightarrow 0$.

of $x$ by a nilpotent Lie algebra.

Suppose that $V$ is a finite-dimensional $R$-module. Define the continuous cohomo$\operatorname{logy}$ of $\mathfrak{u}$ and $\mathfrak{g}$ with coefficients in $V$ to be the direct limit of the cohomology of their canonical finite dimensional quotients:

$$
H_{\text {cts }}^{\bullet}(\mathfrak{l})=\underset{\mathfrak{n}}{\lim } H^{\bullet}(\mathfrak{u} / \mathfrak{n})
$$

and

$$
H_{\text {cts }}^{\bullet}(\mathfrak{g}, V)=\underset{\mathfrak{l}}{\lim } H^{\bullet}(\mathfrak{g} / \mathfrak{n}, V) \text { and } H_{\text {cts }}^{\bullet}(\mathfrak{l}, V)=\underset{\mathfrak{l}}{\lim } H^{\bullet}(\mathfrak{l} / \mathfrak{n}, V)=H_{\text {cts }}^{\bullet}(\mathfrak{l t}) \otimes V .
$$

These can be computed using continuous cochains; the group $H_{\mathrm{cts}}^{\bullet}(\mathfrak{g}, V)$ is the cohomology of the complex

$$
\operatorname{Hom}^{\text {cts }}\left(\Lambda^{\bullet} \mathfrak{g}, V\right):=\underset{\mathfrak{n}}{\lim } \operatorname{Hom}\left(\Lambda^{\bullet}(\mathfrak{g} / \mathfrak{n}), V\right)
$$

of continuous cochains. The differential is induced by the dual of the bracket. A complex which computes $H_{\mathrm{cts}}^{\bullet}(\mathfrak{l}, V)$ is obtained by replacing $\mathfrak{g}$ by $\mathfrak{u}$.

Since the Lie algebras $\mathfrak{g}$ of $\mathcal{G}$ and $\mathfrak{u}$ of $\mathcal{U}$ are inverse limits of the Lie algebras of the finite dimensional quotients of $\mathcal{G}$ and $\mathcal{U}$, respectively, it follows from Theorem 3.12 that each has a natural weight filtration. Since the category of $\mathcal{G}$-modules is Abelian, these pass to $H_{\mathrm{cts}}^{\bullet}(\mathfrak{l t})$.

PROPOSITION 4.5. The Lie algebras $\mathfrak{g}$ of $\mathcal{G}, \mathfrak{r}$ of $R$ and $\mathfrak{u}$ of $\mathcal{U}$ have natural weight filtrations. These satisfy

$$
\mathfrak{g}=W_{0} \mathfrak{g}, \quad W_{n} \mathfrak{u}=W_{n} \mathfrak{g} \text { whenever } n<0 .
$$

These weight filtrations pass to cohomology. In particular, each cohomology group $H_{\mathrm{cts}}^{m}(\mathfrak{u l})$ has a canonical weight filtration which satisfies

$$
\mathrm{Gr}_{m}^{W} H_{\mathrm{cts}}^{n}(\mathfrak{l t})=0 \text { if } m<n .
$$

More precisely, if $W_{m-1} H_{\mathrm{cts}}^{1}(\mathfrak{l t})=0$, then $W_{n m-1} H_{\mathrm{cts}}^{n}(\mathfrak{i t})=0$.

Proof. We need only prove the last statement. Since $H_{\text {cts }}^{1}(\mathfrak{u})$ has weights $\geqslant m, \mathfrak{t}$ has weights $\leqslant-m$, and its continuous dual $\mathfrak{u}^{*}$ (a direct limit of finite-dimensional $\mathcal{G}$-modules) has weights $\geqslant m$. It follows that the space

$$
\operatorname{Hom}^{\text {cts }}\left(\Lambda^{\bullet} \mathfrak{u}, k\right)
$$

of degree $n$ continuous cochains on $\mathfrak{u}$ has weights $\geqslant n m$. Since the bracket is a morphism, the Chevalley-Eilenberg complex

$$
\left(\operatorname{Hom}^{\mathrm{cts}}\left(\Lambda^{\bullet} \mathfrak{u}, k\right),-[,]^{*}\right)
$$

of continuous cochains on $\mathfrak{t}$ is a complex of $\mathcal{G}$-modules. Its cohomology is therefore a graded $\mathcal{G}$-module and thus has a weight filtration induced from that of $\mathfrak{u}$. Since 
the $n$-cochains have weights $\geqslant n m$, it follows that the weights on $H^{n}(\mathfrak{u})$ are also $\geqslant n m$.

\subsection{BASIC STRUCTURE OF $\mathfrak{g}$ AND $\mathfrak{u}$}

Each finite dimensional representation $V$ of $R$ can be decomposed $V=\bigoplus_{n \in Z} V_{n}$ under the $\mathrm{G}_{m}$ action. We shall call $V$ pure of weight $n$ if $V=V_{n}$, and negatively weighted if $V_{n}=0$ for all $n \geqslant 0$. Since $w$ is central, each $V_{n}$ is an $R$-module. Thus, if $V$ is indecomposable (i.e., cannot be written as a direct sum of two nontrivial submodules), $V$ is pure of some weight we shall denote by $n(V)$.

Denote by $H_{\mathrm{cts}}^{i}(\Gamma, V)$ the cohomology of the complex of continuous cochains

$$
f: \Gamma^{m+1} \rightarrow V
$$

where $V$ is viewed as a continuous $\Gamma$-module via $\rho: \Gamma \rightarrow R\left(Q_{\ell}\right)$. This is the same notion of continuous cohomology as used in [34, Sect. 2].

THEOREM 4.6. For all pure $R$-modules $V$, there are natural isomorphisms

$$
\operatorname{Hom}_{R}^{\text {cts }}\left(H_{1}(\mathcal{U}), V\right) \cong \begin{cases}H_{\text {cts }}^{1}(\Gamma, V) & \text { when } n(V)<0 \\ 0 & \text { when } n(V) \geqslant 0\end{cases}
$$

and

$$
\operatorname{Hom}_{R}^{\text {cts }}\left(\operatorname{Gr}_{n}^{W} H_{1}(\mathcal{U}), V\right) \cong \begin{cases}H_{\text {cts }}^{1}(\Gamma, V) & \text { when } n=n(V)<0 \\ 0 & \text { otherwise. }\end{cases}
$$

The second assertion follows directly from the first, which is proved in Section 5.

COROLLARY 4.7. If $H_{\mathrm{cts}}^{1}(\Gamma, V)$ vanishes for all pure representations $V$ of $R$ of negative weight, then the weighted completion of $\Gamma$ relative to $\rho: \Gamma \rightarrow R$ is $\rho$ itself.

Proof. The theorem above implies that $H_{1}(\mathfrak{t})=0$. Since $\mathfrak{u}$ is pronilpotent, $\mathfrak{u}$ is trivial.

\subsection{THE CASE $R$ REDUCTIVE}

Suppose now that $R$ is reductive. Let $\left\{V_{\alpha}\right\}_{\alpha}$ be a set of representatives of the isomorphism classes of finite-dimensional irreducible representations of $R$. For convenience, we set $n(\alpha)=n\left(V_{\alpha}\right)$.

The next result follows directly from Theorem 4.6.

THEOREM 4.8. If $H_{\mathrm{cts}}^{1}\left(\Gamma, V_{\alpha}\right)$ is finite-dimensional for all $\alpha$ with $n(\alpha)<0$, then

$$
H_{\mathrm{cts}}^{1}(\mathfrak{l t}) \cong \bigoplus_{\{\alpha: n(\alpha) \geqslant 1\}} H_{\mathrm{cts}}^{1}\left(\Gamma, V_{\alpha}^{*}\right) \otimes V_{\alpha}
$$


and

$$
H_{1}(\mathcal{U}) \cong H_{1}(\mathfrak{H}) \cong \underset{F}{\lim } \bigoplus_{\alpha \in F} H_{\mathrm{cts}}^{1}\left(\Gamma, V_{\alpha}\right)^{*} \otimes V_{\alpha}
$$

where $F$ ranges over the finite subsets of $\{\alpha: n(\alpha)<0\}$. More precisely, $W_{0} H_{\mathrm{cts}}^{1}(\mathfrak{1})$ vanishes and, if $m>0$, then

$$
\mathrm{Gr}_{m}^{W} H_{\mathrm{cts}}^{1}(\mathfrak{H}) \cong \bigoplus_{\{\alpha: n(\alpha)=m\}} H_{\mathrm{cts}}^{1}\left(\Gamma, V_{\alpha}^{*}\right) \otimes V_{\alpha}
$$

In the reductive case, we are able to control $H_{\mathrm{cts}}^{2}(\mathfrak{H})$ as well, which will allow us to control the relations in $\mathfrak{u}$.

THEOREM 4.9. There is a natural injective R-invariant homomorphism

$$
\Phi: H_{\mathrm{cts}}^{2}(\mathfrak{u}) \hookrightarrow \bigoplus_{\{\alpha: n(\alpha) \geqslant 2\}} H_{\mathrm{cts}}^{2}\left(\Gamma, V_{\alpha}^{*}\right) \otimes V_{\alpha} .
$$

In addition, if $m>1$, then

$$
\mathrm{Gr}_{m}^{W} H_{\mathrm{cts}}^{2}(\mathfrak{u}) \subseteq \bigoplus_{\{\alpha: n(\alpha)=m\}} H_{\mathrm{cts}}^{2}\left(\Gamma, V_{\alpha}^{*}\right) \otimes V_{\alpha}
$$

The proof is given in Section 6.

COROLLARY 4.10. Suppose that $H_{\mathrm{cts}}^{1}\left(\Gamma, V_{\alpha}^{*}\right)$ vanishes for all $\alpha$ satisfying $0<n(\alpha)$ $<d$. If $H_{\mathrm{cts}}^{2}\left(\Gamma, V_{\alpha}^{*}\right)$ vanishes for all $\alpha$ with $n(\alpha) \geqslant 2 d$, then $\mathrm{Gr}_{\bullet}^{W} \mathfrak{u}$ is a free Lie algebra. Moreover, any lift of a basis of $\mathrm{Gr}_{\bullet}^{W} H_{1}(\mathfrak{u})$ to $\mathrm{Gr}_{\bullet}^{W} \mathfrak{u}$ is a free generating set.

Proof. The condition on $H_{\mathrm{cts}}^{1}\left(\Gamma, V_{\alpha}^{*}\right)$ implies, by Theorem 4.9 , that $W_{d-1}$ $H_{\mathrm{cts}}^{1}(\mathfrak{u})=0$. The last assertion in Proposition 4.5 implies that $W_{2 d-1} H_{\mathrm{cts}}^{2}(\mathfrak{u})=0$. The assumption about $H_{\text {cts }}^{2}\left(\Gamma, V_{\alpha}^{*}\right)$ and the last assertion of Theorem 4.9 imply that

$$
\mathrm{Gr}_{m}^{W} H_{\mathrm{cts}}^{2}(\mathfrak{u})=0
$$

when $m \geqslant 2 d$, so that $\mathrm{Gr}_{\bullet}^{W} H_{\text {cts }}^{2}(\mathfrak{l t})=0$. Since $\mathrm{Gr}_{\bullet}^{W}$ is an exact functor, it commutes with homology. So it follows that $H^{2}\left(\mathrm{Gr}_{\bullet}^{W} \mathfrak{u}\right)$ vanishes. The result now follows from the following lemma.

LEMMA 4.11. If $\mathfrak{n}=\bigoplus_{m<0} \mathfrak{n}_{m}$ is a graded Lie algebra over a field of characteristic zero, then $\mathfrak{n}$ is free if and only if $H^{2}(\mathfrak{n})=0$.

Proof. By Corollary 2.2, there is a free graded Lie algebra $\mathfrak{f}=\bigoplus_{m<0} \tilde{f}_{m}$ and a graded Lie algebra homomorphism $\mathfrak{\uparrow} \rightarrow \mathfrak{n}$ which is surjective and induces an isomorphism on $H_{1}$. Denote the kernel of this by $\mathfrak{a}$. Note that $\mathfrak{n}$ is free if and only if $a=0$. Since $a$ is an ideal in a negatively graded Lie algebra, $a=0$ if and only if $a /[\mathfrak{f}, \mathfrak{a}]=0$. 
There is a spectral sequence

$$
E_{2}^{a, b}=H^{a}\left(\mathfrak{n}, H^{b}(\mathfrak{a})\right) \Rightarrow H^{a+b}(\mathfrak{f}) .
$$

Since $\mathfrak{f}$ is free, $H^{2}(\mathfrak{f})=0$. It follows that

$$
d_{2}: H^{0}\left(\mathfrak{n}, H^{1}(\mathfrak{a})\right) \rightarrow H^{2}(\mathfrak{n})
$$

is an isomorphism. The result follows as $H^{0}\left(\mathfrak{n}, H^{1}(\mathfrak{a})\right)$ is the graded dual of $\mathfrak{a} /[\mathfrak{f}, \mathfrak{a}]$.

EXAMPLE 4.12. Suppose that $\Gamma=Z_{\ell}^{\times}$, that $R=\mathrm{G}_{m / Q_{\ell}}$ and that $\rho: Z_{\ell}^{\times} \hookrightarrow$ $\mathrm{G}_{m}\left(\mathrm{Q}_{\ell}\right)=\mathrm{Q}_{\ell}^{\times}$is the natural inclusion. Take $w$ to be the inverse of the square of the standard character. ${ }^{\star}$ In this example we compute the weighted completion of $Z_{\ell}^{\times}$ with respect to $\rho$ and $w$. Note that

$$
H_{\mathrm{cts}}^{1}\left(Z_{\ell}^{\times}, Q^{m}\right)=0
$$

for all non-zero $m \in Z$. This can be seen as follows.

For each $a \in Z_{\ell}^{\times}$, the mapping

$$
\text { a: } Q^{m} \rightarrow Q^{m} \quad x \mapsto a^{m} x
$$

is $Z_{\ell}^{\times}$-equivariant, and thus induces an automorphism of $H^{n}\left(Z_{\ell}^{\times}, Q^{m}\right)$. A general fact in group cohomology implies that this automorphism is trivial. (cf. [28, p. 116].) It follows that $\left(1-a^{m}\right)$ annihilates $H^{n}\left(Z_{\ell}^{\times}, Q^{m}\right)$ for all $a \in Z_{\ell}^{\times}$. But if $m \neq 0$ and $a \neq 1$, then $\left(1-a^{m}\right)$ is in $\mathrm{Q}_{\ell}^{\times}$. It follows that $H^{n}\left(Z_{\ell}^{\times}, Q^{m}\right)$ vanishes when $m \neq 0$.

Theorem 4.6 tells us that the unipotent radical $\mathcal{U}$ of the weighted completion of $Z_{\ell}^{\times}$ is trivial, so that the weighted completion of $Z_{\ell}^{\times}$with respect to $\rho$ is just $\rho: Z_{\ell}^{\times} \rightarrow \mathrm{G}_{m}\left(\mathrm{Q}_{\ell}\right)$.

More generally, we can take $\Gamma$ in the previous example to be any open subgroup of $Z_{\ell}^{\times}$. One has the same vanishing of cohomology and consequently, that the weighted completion of $\Gamma$ with respect to $\rho: \Gamma \rightarrow \mathrm{G}_{m}\left(Q_{\ell}\right)$ is just $\rho$ itself.

\subsection{NATURALITY}

Here we record some more technical naturality statements that are needed in the sequel.

Suppose that $f: \Gamma_{1} \rightarrow \Gamma_{2}$ is a continuous homomorphism between two profinite groups. Suppose that $R_{1}$ and $R_{2}$ are two algebraic $\mathbb{Q}_{\ell}$-groups with central cocharac-

${ }^{\star}$ We use the inverse of the square of the standard character so that later, when considering the weighted completion of $\pi_{1}(\operatorname{Spec} Z[1 / \ell])$, for example, the representation theoretic weights agree with weights defined using Frobenius. This square makes no difference to the weighted completion, it just doubles the indexing of the weight filtration. 
ters $w_{1}$ and $w_{2}$, respectively. Suppose that

$$
\rho_{1}: \Gamma_{1} \rightarrow R_{1}\left(Q_{\ell}\right) \text { and } \rho_{2}: \Gamma_{2} \rightarrow R_{2}\left(Q_{\ell}\right)
$$

are two continuous, Zariski dense homomorphisms. We can form the weighted completions $\mathcal{G}_{1}$ and $\mathcal{G}_{2}$ of $\Gamma_{1}$ and $\Gamma_{2}$. Suppose that $\phi: R_{1} \rightarrow R_{2}$ is a homomorphism of $Q_{\ell}$-groups such that

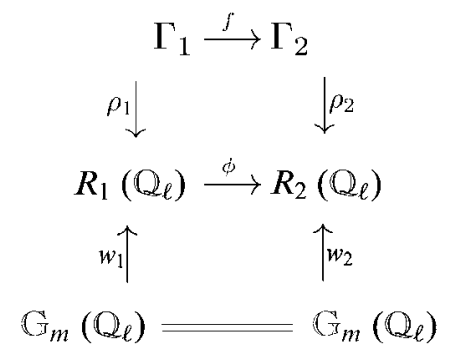

commutes.

The following result is easily proved using the universal mapping property of weighted completion and Theorem 3.12.

PROPOSITION 4.13. Under these hypotheses, there is a homomorphism $\Phi: \mathcal{G}_{1} \rightarrow \mathcal{G}_{2}$ of proalgebraic $\mathrm{Q}_{\ell}$-groups such that the diagram

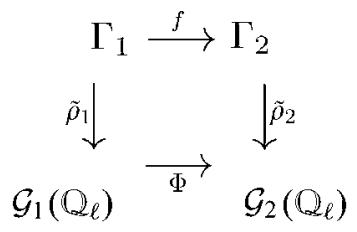

commutes. Moreover, the induced homomorphism $\mathfrak{g}_{1} \rightarrow \mathfrak{g}_{2}$ on Lie algebras preserves the weight filtration and is strictly compatible with it.

We are now in the situation described in Paragraph 3.3 and can thus apply Theorem 3.12 .

\subsection{RIGHT EXACTNESS}

Our goal in this paragraph is to understand the relation between the kernel of $\mathcal{G} \rightarrow R$ and the $\ell$-adic unipotent completion of the kernel of $\rho: \Gamma \rightarrow R\left(Q_{\ell}\right)$.

We continue with the notation from the beginning of this section: $\Gamma$ is a profinite group, $\rho: \Gamma \rightarrow R\left(Q_{\ell}\right)$ is a continuous, Zariski dense homomorphism from $\Gamma$ to an algebraic $Q_{\ell}$-group, $w$ is a central cocharacter of $R, \mathcal{G}$ is the corresponding weighted completion of $\Gamma$, and $\mathcal{U}$ is the kernel of $\mathcal{G} \rightarrow R$. 
Denote by $\mathcal{R}$ the weighted completion of $\operatorname{im} \rho$ with respect to the inclusion $\operatorname{im} \rho \hookrightarrow R\left(Q_{\ell}\right)$ and $w$. The homomorphisms $\Gamma \rightarrow \operatorname{im} \rho$ and $\operatorname{ker} \rho \rightarrow \mathcal{U}\left(\mathrm{Q}_{\ell}\right)$ induce homomorphisms

$$
\mathcal{G} \rightarrow \mathcal{R} \quad \text { and } \quad(\operatorname{ker} \rho)_{/ Q_{\ell}}^{\text {un }} \rightarrow \mathcal{U}
$$

where $(\operatorname{ker} \rho)_{/ Q_{\ell}}^{\text {un }}$ denotes the $\ell$-adic unipotent completion (Paragraph A.2) of ker $\rho$.

PROPOSITION 4.14. The sequence

$$
(\operatorname{ker} \rho)_{/ Q_{\ell}}^{\text {un }} \rightarrow \mathcal{G} \rightarrow \mathcal{R} \rightarrow 1
$$

is exact.

Proof. Since $\Gamma \rightarrow \mathcal{R}\left(Q_{\ell}\right)$ is Zariski dense, $\mathcal{G} \rightarrow \mathcal{R}$ is surjective. Denote the image of $(\operatorname{ker} \rho)_{/ Q_{\ell}}^{\mathrm{un}}$ in $\mathcal{U}$ by $\mathcal{K}$. Since the diagram

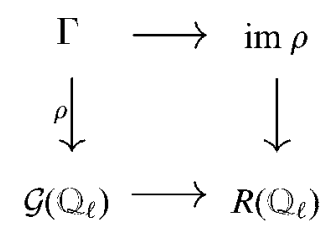

commutes, the image of $\operatorname{ker} \rho$ in $\mathcal{R}\left(Q_{\ell}\right)$ is trivial. It follows that $\mathcal{K}$ is contained in the kernel of $\mathcal{G} \rightarrow \mathcal{R}$ and that the composite

$$
(\operatorname{ker} \rho)_{/ Q_{\ell}}^{\text {un }} \rightarrow \mathcal{G} \rightarrow \mathcal{R}
$$

is trivial. It remains to show that $\mathcal{K}$ contains the kernel of $\mathcal{G} \rightarrow \mathcal{R}$.

Note that $\mathcal{K}$ is the Zariski closure of the image of $\operatorname{ker} \rho$ in $\mathcal{U}$. Since $\operatorname{ker} \rho$ is normal in $\Gamma$, and since $\Gamma$ is Zariski dense in $\mathcal{G}, \mathcal{K}$ is normal in $\mathcal{G}$. The homomorphism $\rho$ induces a homomorphism $\operatorname{im} \rho \rightarrow(\mathcal{G} / \mathcal{K})\left(Q_{\ell}\right)$. Because $\mathcal{G} / \mathcal{K}$ is a negatively weighted extension of $R$, the universal mapping property of $\mathcal{R}$ gives a splitting $\mathcal{R} \rightarrow \mathcal{G} / \mathcal{K}$ of $\mathcal{G} \rightarrow \mathcal{R}$. Since the image of $\Gamma$ is Zariski dense in $\mathcal{G}, \operatorname{im} \rho$ has Zariski dense image in $\mathcal{G} / \mathcal{K}$, which implies that this splitting is surjective, and therefore an isomorphism $\mathcal{R} \rightarrow \mathcal{G} / \mathcal{K}$.

COROLLARY 4.15. If $H_{\mathrm{cts}}^{1}\left(\mathrm{im} \rho, V_{\alpha}\right)$ vanishes for all irreducible representations $V_{\alpha}$ of $R$ for which $n(\alpha)<0$, then the sequence

$$
(\operatorname{ker} \rho)_{/ Q_{\ell}}^{\text {un }} \rightarrow \mathcal{G} \rightarrow R \rightarrow 1
$$

is exact. In particular, the image of $\operatorname{ker} \rho$ in $\mathcal{U}\left(Q_{\ell}\right)$ is Zariski dense.

Proof. Corollary 4.7 implies that $R=\mathcal{R}$. The result follows.

\section{Proof of Theorem 4.6}

The proof is an exercise in group cohomology. We continue with the notation from Section 4, except that we will abuse notation and not distinguish between an 
algebraic $\mathbb{Q}_{\ell}$-group and the group of its $Q_{\ell}$ rational points.

Recall that

$$
\mathcal{U}=\lim _{\overleftarrow{\hat{\rho}}} U_{\hat{\rho}}
$$

where $\hat{\rho}$ ranges over all continuous, Zariski dense representations $\hat{\rho}: \Gamma \rightarrow G_{\hat{\rho}}\left(Q_{\ell}\right)$ into a negatively weighted extension $G_{\hat{\rho}}$ of $R$ by a unipotent group, which we shall denote by $U_{\hat{\rho}}$.

PROPOSITION 5.1. There are natural isomorphisms

$$
H_{1}(\mathfrak{u})=H_{1}(\mathcal{U})=\lim _{\overleftarrow{\hat{\rho}}} H_{1}\left(U_{\hat{\rho}}\right) \quad \text { and } \quad H_{\text {cts }}^{1}(\mathfrak{i})=\lim _{\overrightarrow{\hat{\rho}}} H^{1}\left(\mathfrak{l} \hat{\rho}_{\hat{\rho}}\right)
$$

Each finite-dimensional $R$-module will be considered as a continuous $\Gamma$-module via $\rho$. Note that there are natural one-to-one correspondences between:

(i) the set of continuous 1-cocycles $f: \Gamma \rightarrow V$;

(ii) the set of continuous homomorphisms $\phi: \Gamma \rightarrow R \ltimes V$ that lift $\rho$;

(iii) the set of continuous splittings $s: \Gamma \rightarrow \Gamma \ltimes V$ of the extension

$$
0 \rightarrow V \rightarrow \Gamma \times V \rightarrow \Gamma \rightarrow 1 .
$$

Under this correspondence, cocycle $f$, the lift $\gamma \mapsto(\rho(\gamma), f(\gamma))$, and the splitting $\gamma \mapsto(\gamma, f(\gamma))$ all correspond.

The following result is somewhat standard and is easily proved. It is a basic tool in the proof of Theorem 4.6.

LEMMA 5.2. There are natural one-to-one correspondences between the following three sets:

$$
\begin{aligned}
& \left\{\begin{array}{l}
\text { continuous homomorphisms } \\
\phi: \Gamma \rightarrow R \times V \text { that lift } \rho
\end{array}\right\} / \begin{array}{c}
\text { conjugation by } \\
\text { elements of } V
\end{array} \\
& \left\{\begin{array}{c}
\text { continuous splittings s: } \\
\Gamma \rightarrow \Gamma \times V \text { of } \Gamma \times V \longrightarrow \Gamma
\end{array}\right\} / \begin{array}{c}
\text { conjugation by } \\
\text { elements of } V
\end{array}
\end{aligned}
$$

and $H_{\mathrm{cts}}^{1}(\Gamma, V)$. The bijections are induced by the bijections above.

We now prove the Theorem. First, if $V$ is a pure representation of $R$ with $n(V) \geqslant 0$, then $\operatorname{Hom}_{S}^{\mathrm{cts}}\left(H_{1}(\mathcal{U}), V\right)=0$ as $H_{1}(\mathcal{U})$ is negatively weighted. So suppose that $V$ is a pure representation of negative weight. In this case, the extension $S \ltimes V$ is negatively weighted.

Each element of $H_{\mathrm{cts}}^{1}(\Gamma, V)$ gives a homomorphism $\hat{\rho}: \Gamma \rightarrow R \times V$ that lifts $\rho$. By the universal mapping property of $\mathcal{G}$, there is a homomorphism $\theta: \mathcal{G} \rightarrow R \times V$ whose composition with the canonical mapping $\Gamma \rightarrow \mathcal{G}\left(Q_{\ell}\right)$ is $\hat{\rho}$. Since $R \times V$ is an algebraic group, this homomorphism induces a continuous mapping $H_{1}(\mathcal{U}) \rightarrow V$, which is 
clearly $R$-invariant. This homomorphism depends only on the homomorphism $\hat{\rho}$ up to conjugation by elements of $V$. There is thus a well defined homomorphism

$$
H_{\mathrm{cts}}^{1}(\Gamma, V) \rightarrow \operatorname{Hom}_{R}^{\mathrm{cts}}\left(H_{1}(\mathcal{U}), V\right) .
$$

Conversely, suppose that $\phi: H_{1}(\mathcal{U}) \rightarrow V$ is continuous and $R$-invariant. Pushing out the extension

$$
0 \rightarrow H_{1}(\mathcal{U}) \rightarrow \mathcal{G} /[\mathcal{U}, \mathcal{U}] \rightarrow R \rightarrow 1
$$

along $\phi$, we obtain an extension

$$
0 \rightarrow V \rightarrow G_{\phi} \rightarrow R \rightarrow 1
$$

which is algebraic as $\phi$ is continuous. Composing the canonical mapping $\Gamma \rightarrow \mathcal{G}$ with $\mathcal{G} \rightarrow \mathcal{G} /[\mathcal{U}, \mathcal{U}] \rightarrow G_{\phi}$, we obtain a homomorphism $\Gamma \rightarrow G_{\phi}$ which lifts $\rho$. By Lemma 3.7, the projection $G_{\phi} \rightarrow R$ splits, and the splitting is unique up to conjugation by an element of $V$. Thus $\phi$ induces a homomorphism $\Gamma \rightarrow R \ltimes V$ which is unique up to conjugation by an element of $V$, and therefore an element of $H_{\mathrm{cts}}^{1}(\Gamma, V)$. This mapping is easily seen to be the inverse of the one above. This completes the proof of Theorem 4.6.

\section{Proof of Theorem 4.9}

\subsection{A TECHNICAL LEMMA}

Suppose that $R$ is a reductive group over a field $k$ of characteristic zero. Suppose that $\mathfrak{n}$ is a nilpotent Lie algebra over $k$ and that $R$ acts on $\mathfrak{n}$ as a group of automorphisms.

Suppose that $V$ is a finite-dimensional $R$-module. Each element of $\theta \in H_{\text {cts }}^{2}(\mathfrak{n}) \otimes V$ determines a central extension

$$
0 \rightarrow V \rightarrow \tilde{\mathfrak{n}}_{\theta} \rightarrow \mathfrak{n} \rightarrow 0 .
$$

LEMMA 6.1. The action of $R$ on $\mathfrak{n}$ and $V$ lifts to an action on $\tilde{\mathfrak{n}}_{\theta}$ as a group of Lie algebra automorphisms if and only if $\theta \in\left[H_{\mathrm{cts}}^{2}(\mathfrak{n}) \otimes V\right]^{G}$.

Proof. We will prove the sufficiency of the condition; necessity is left as an exercise.

Since $R$ is reductive and acts on the finite-dimensional complex

$$
C^{\bullet}(\mathfrak{n}):=\left(\Lambda^{\bullet} \mathfrak{n}^{*},-[,]^{*}\right)
$$

of Lie algebra cochains,

$$
\left[H_{\mathrm{cts}}^{\bullet}(\mathfrak{n}) \otimes V\right]^{R}=H^{\bullet}\left(\left[C^{\bullet}(\mathfrak{n}) \otimes V\right]^{R}\right) .
$$

In particular, each

$$
\theta \in\left[H_{\mathrm{cts}}^{2}(\mathfrak{l}) \otimes V\right]^{R}
$$


can be represented by a continuous $R$-invariant cocycle $\Theta: \Lambda^{2} \mathfrak{n} \rightarrow V$. The Lie algebra $\tilde{\mathfrak{n}}_{\theta}$ can be constructed as $\mathfrak{n} \oplus V$ with bracket

$$
[(x, v),(y, w)]=([x, y], \Theta(x \wedge y)) .
$$

The obvious action of $R$ on $\mathfrak{n} \oplus V$ preserves the bracket as $\Theta$ is $R$ invariant.

\subsection{PROOF OF THE THEOREM}

We continue with the notation from Section 4. Suppose in addition that $R$ is reductive. Let $\left\{V_{\alpha}\right\}$ be a set of representatives of the isomorphism classes of the finite-dimensional, irreducible $R$-modules. It suffices to construct an injective linear map

$$
\Phi_{\alpha}:\left[H_{\mathrm{cts}}^{2}(\mathfrak{u}) \otimes V_{\alpha}\right]^{R} \rightarrow H_{\mathrm{cts}}^{2}\left(\Gamma, V_{\alpha}\right) .
$$

Suppose that $\theta \in\left[H_{\mathrm{cts}}^{2}(\mathfrak{H}) \otimes V_{\alpha}\right]^{R}$. Then there exists an algebraic quotient $G$ of $\mathcal{G}$ which is an extension

$$
1 \rightarrow N \rightarrow G \rightarrow R \rightarrow 1
$$

where $N$ is unipotent, and a class $\tilde{\theta} \in\left[H^{2}(\mathfrak{n}) \otimes V_{\alpha}\right]^{R}$, where $\mathfrak{n}$ is the Lie algebra of $N$, whose image under $H^{2}(\mathfrak{n}) \rightarrow H_{\text {cts }}^{2}(\mathfrak{l l})$ is $\theta$. The class $\tilde{\theta}$ determines a central extension

$$
0 \rightarrow V_{\alpha} \rightarrow \tilde{N}_{\theta} \rightarrow N \rightarrow 1 \text {. }
$$

Choose a Levi decomposition

$$
G \cong R \times N .
$$

This determines an action of $R$ on $\mathfrak{n}$. Lemma 6.1 implies that the $R$ action on $N$ lifts to $\tilde{N}_{\theta}$. We can thus form the extension

$$
0 \rightarrow V_{\alpha} \rightarrow R \ltimes \tilde{N}_{\theta} \rightarrow R \ltimes N \rightarrow 1 .
$$

Pulling this extension back along the quotient mapping $\mathcal{G} \rightarrow G=R \ltimes N$, we obtain an extension

$$
0 \rightarrow V_{\alpha} \rightarrow \tilde{\mathcal{G}}_{\theta} \rightarrow \mathcal{G} \rightarrow 1
$$

of proalgebraic groups. Pulling this extension back along $\Gamma \rightarrow \mathcal{G}$, we obtain an extension

$$
0 \rightarrow V_{\alpha} \rightarrow \tilde{\Gamma}_{\theta} \rightarrow \Gamma \rightarrow 1 .
$$

This gives a class in $H_{\mathrm{cts}}^{2}\left(\Gamma, V_{\alpha}\right)$. This class is easily seen to depend only on the class of $\theta$. This procedure therefore defines a map

$$
\Phi_{\alpha}:\left[H_{\mathrm{cts}}^{2}(\mathfrak{u}) \otimes V_{\alpha}\right]^{R} \rightarrow H_{\mathrm{cts}}^{2}\left(\Gamma, V_{\alpha}\right) .
$$


If $\Phi_{\alpha}(\theta)$ vanishes, the extension (5) splits. The universal mapping property of $\Gamma \rightarrow \mathcal{G}\left(Q_{\ell}\right)$ then implies that (4) splits. This restricts to give a splitting of the extension

$$
0 \rightarrow V_{\alpha} \rightarrow \tilde{\mathfrak{u}}_{\theta} \rightarrow \mathfrak{u} \rightarrow 0,
$$

where $\tilde{\mathfrak{H}}_{\theta}$ denotes the Lie algebra of the unipotent radical of $\tilde{\mathcal{G}}_{\theta}$. It follows that $\theta$ vanishes.

\section{The Weighted Completion of $\pi_{1}\left(\operatorname{Spec} \mathcal{O}_{F, S}\right)$}

Fix a prime number $\ell$. Suppose that $F$ is a number field. Denote its ring of integers by $\mathcal{O}_{F}$. Suppose that $S$ is a finite set of closed points of Spec $\mathcal{O}_{F}$ containing all primes over $\ell$. Let $\mathcal{O}_{F, S}$ be the set of $S$-integers of $F$, so that $\operatorname{Spec} \mathcal{O}_{F, S}=\operatorname{Spec} \mathcal{O}_{F}-S$. Set

$$
G_{F, S}=\pi_{1}\left(\operatorname{Spec} \mathcal{O}_{F, S}, \operatorname{Spec} \bar{F}\right) \text {. }
$$

This is the Galois group of the maximal algebraic extension of $F$ unramified outside $S$.

One has the $\ell$-adic cyclotomic character $\chi_{\ell}: G_{F, S} \rightarrow Z_{\ell}^{\times}$. Its image is an open subgroup of $Z_{\ell}^{\times}$and hence of finite index. Composing this with the inclusion $Z_{\ell}^{\times} \hookrightarrow \mathrm{G}_{m}\left(Q_{\ell}\right)$, we obtain a continuous homomorphism

$$
\rho_{\ell}: G_{F, S} \rightarrow \mathrm{G}_{m}\left(Q_{\ell}\right)
$$

with Zariski dense image. As in Example 4.12, we take $w: \mathrm{G}_{m} \rightarrow \mathrm{G}_{m}$ be the morphism $x \mapsto x^{-2}$.

Since $\mathrm{G}_{m}$ is reductive, we can form the weighted completion

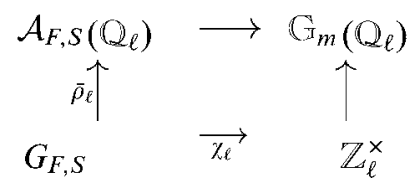

of $G_{F, S}$ with respect to $\rho_{\ell}$. Here $\mathcal{A}_{F, S}$ is a proalgebraic group over $Q_{\ell}$ with reductive quotient $\mathrm{G}_{m}$. Denote the prounipotent radical of $\mathcal{A}_{F, S}$ by $\mathcal{K}_{F, S}$ and the Lie algebras of $\mathcal{A}_{F, S}$ and $\mathcal{K}_{F, S}$ by $\mathfrak{a}_{F, S}$ and $\mathfrak{f}_{F, S}$, respectively.

\section{PROPOSITION 7.1. The homomorphism}

$$
\operatorname{ker}\left\{\rho_{\ell}: G_{F, S} \rightarrow \mathrm{Gr}_{m}\left(Q_{\ell}\right)\right\} \rightarrow \mathcal{K}_{F, S}\left(Q_{\ell}\right)
$$

induced by $\tilde{\rho}_{\ell}: G_{F, S} \rightarrow \mathcal{A}_{F, S}\left(Q_{\ell}\right)$ has Zariski dense image.

Proof. This follows directly from Example 4.12 and Corollary 4.15 as the image of $\chi_{\ell}: G_{F, S} \rightarrow Z_{\ell}^{\times}$is open.

\subsection{BASIC STRUCTURE OF $\mathfrak{a}_{F, S}$}

The Lie algebra $\mathfrak{a}_{F, S}$, being the Lie algebra of a weighted completion, has a natural weight filtration. Note that since $w$ is the inverse of the square of the standard character, all weights are even. Thus the weight filtration of $\mathfrak{a}_{F, S}$ satisfies

$$
\mathfrak{a}_{F, S}=W_{0} \mathfrak{a}_{F, S}, \quad \mathfrak{f}_{F, S}=W_{-2} \mathfrak{a}_{F, S} \quad \text { and } \quad \mathrm{Gr}_{2 n+1}^{W} \mathfrak{a}_{F, S}=0 \text { for all } n .
$$


THEOREM 7.2. The Lie algebra $\mathrm{Gr}_{\bullet}^{W} \mathfrak{f}_{F, S}$ is a free Lie algebra and there is a natural $\mathrm{G}_{m}$-equivariant isomorphism

$$
H_{\mathrm{cts}}^{1}\left(\mathfrak{f}_{F, S}\right) \cong \bigoplus_{n=1}^{\infty} H_{\mathrm{cts}}^{1}\left(G_{F, S}, Q_{\ell}(n)\right) \otimes Q_{\ell}(-n) \cong \bigoplus_{n=1}^{\infty} Q_{\ell}(-n)^{d_{n}},
$$

where

$$
d_{n}= \begin{cases}r_{1}+r_{2}+\# S-1, & \text { when } n=1 ; \\ r_{1}+r_{2}, & \text { when } n \text { is odd and }>1 ; \\ r_{2}, & \text { when } n \text { is even. }\end{cases}
$$

Here $r_{1}$ and $r_{2}$ are the number of real and complex places of $F$, respectively. Moreover, each weight graded quotient of $\mathfrak{a}_{F, S}$ is a finite dimensional $\mathrm{Q}_{\ell}$-vector space. Any lift of a graded basis of $H_{1}\left(\mathrm{Gr}_{\bullet}^{W \mathfrak{f}_{F, S}}\right)$ to a graded set of elements of $\mathrm{Gr}_{\bullet}^{W \mathfrak{f}_{F, S}}$ freely generates $\mathrm{Gr}_{\bullet}^{W} \mathfrak{f}_{F, S}$.

Proof. This follows directly from Theorem 4.8, Corollary 4.10, and Theorem B.1.

7.2. THE CASE $F=\mathrm{Q}$

Set

$$
G_{\ell}=G_{\mathrm{Q},\{\ell\}}, \mathfrak{a}_{\ell}=\mathfrak{a}_{\mathrm{Q},\{\ell\}}, \mathfrak{f}_{\ell}=\mathfrak{f}_{\mathrm{Q},\{\ell\}}, \mathcal{A}_{\ell}=\mathcal{A}_{\mathrm{Q},\{\ell\}}, \mathcal{K}_{\ell}=\mathcal{K}_{\mathrm{Q},\{\ell\}} .
$$

Combining the results of the previous paragraph, we have:

THEOREM 7.3. The Lie algebra $\mathfrak{f}_{\ell}$ is free and

$$
H_{\mathrm{cts}}^{1}\left(\mathfrak{f}_{\ell}\right)=\bigoplus_{n \geqslant 0} \mathrm{Q}_{\ell}(-2 n-1) .
$$

Moreover, there are elements $\sigma_{m} \in W_{-2 m} \mathfrak{a}_{\ell}$ for each odd positive number $m$ whose images in $\mathrm{Gr}_{\bullet}^{W} \mathfrak{\mathfrak { f }}_{\ell}$ generate it freely.

\subsection{THE WEIGHT FILTRATION OF $G_{F S}$}

The weight filtration of $\mathfrak{a}_{F, S}$ can be exponentiated to give a weight filtration

$$
\mathcal{A}_{F, S}=W_{0} \mathcal{A}_{F, S} \supseteq W_{-1} \mathcal{A}_{F, S} \supseteq W_{-2} \mathcal{A}_{F, S} \supseteq W_{-3} \mathcal{A}_{F, S} \supseteq \cdots
$$

of $\mathcal{A}_{F, S}$ whose odd weight graded quotients vanish. This induces a filtration on $G_{F, S}$ by pulling back the weight filtration

$$
G_{F, S}=W_{0} G_{F, S} \supseteq W_{-1} G_{F, S}=W_{-2} G_{F, S} \supseteq W_{-3} G_{F, S}=W_{-4} G_{F, S} \supseteq \cdots
$$

of $\mathcal{A}_{F, S}$ along the natural map $\tilde{\rho}_{\ell}: G_{F, S} \rightarrow \mathcal{A}_{F, S}\left(Q_{\ell}\right)$. Note that $W_{-1} G_{F, S}$ is the kernel of the cyclotomic character. 
For each $m>0$, there is a natural continuous inclusion

$$
\mathrm{Gr}_{-m}^{W} G_{F, S} \hookrightarrow \mathrm{Gr}_{-m}^{W} \mathcal{A}_{F, S}\left(Q_{\ell}\right) \stackrel{\sim}{\longrightarrow} \mathrm{Gr}_{-m}^{W} \mathfrak{a}_{F, S}
$$

THEOREM 7.4. The linear inclusions (6) combine to give an isomorphism

$$
\mathrm{Gr}_{<0}^{W} G_{F, S} \otimes_{Z_{\ell}} Q_{\ell} \hookrightarrow \mathrm{Gr}_{\bullet}^{W \mathfrak{f}_{F, S}}
$$

of $\mathrm{Q}_{\ell}$-Lie algebras.

Since, by Proposition 7.1, the image of ker $\chi_{\ell}$ in $\mathcal{K}_{F, S}\left(Q_{\ell}\right)$ is Zariski dense, this follows from the general lemma proved in the next paragraph.

\subsection{ANOTHER TECHNICAL LEMMA}

Suppose that $\Gamma$ is a profinite group, that $U$ is a prounipotent $Q_{\ell}$-group and that

$$
U=W_{-1} U \supseteq W_{-2} U \supseteq W_{-3} U \supseteq \cdots
$$

is a central filtration of $U$ such that $U / W_{-n} U$ is unipotent for each $n$. Suppose that $\rho: \Gamma \rightarrow U\left(Q_{\ell}\right)$ is a continuous homomorphism. We can induce a filtration

$$
\Gamma=W_{-1} \Gamma \supseteq W_{-2} \Gamma \supseteq W_{-3} \Gamma \supseteq \cdots
$$

on $\Gamma$ by defining $W_{-n} \Gamma=\rho^{-1}\left(W_{-n} U\left(Q_{\ell}\right)\right)$. Note that the mapping

$$
\mathrm{Gr}_{m}^{W} \Gamma \rightarrow \mathrm{Gr}_{m}^{W} U\left(Q_{\ell}\right)
$$

is injective.

LEMMA 7.5. If the image of $\rho: \Gamma \rightarrow U\left(Q_{\ell}\right)$ is Zariski dense, then the inclusion (7) induces an isomorphism

$$
\mathrm{Gr}_{m}^{W} \Gamma \otimes_{Z_{\ell}} Q_{\ell} \rightarrow \mathrm{Gr}_{m}^{W} U\left(Q_{\ell}\right)
$$

for each $m<0$.

Proof. Fix $m<0$. By replacing $U$ by $U / W_{M} U$ for some $M<m$, we may assume that $U$ is unipotent. By replacing $\Gamma$ by $\Gamma / W_{M} \Gamma$, we may assume that $\rho$ is an inclusion.

We will prove the result by induction on the dimension of $U$. First recall that if $U$ is a unipotent group over a field $k$ of characteristic zero, and $V$ is a closed normal subgroup of $U$, also defined over $k$, then the group of $k$-rational points of $U / V$ is isomorphic to $U(k) / V(k)$.

We first consider the case where $U$ is Abelian. In this case, $\Gamma$ is a compact subgroup of $U\left(Q_{\ell}\right) \cong Q_{\ell}{ }^{N}$. Since $Z_{\ell}$ is a PID, and since $\Gamma$ is compact and torsion-free, it is freely generated by $r$ linearly independent elements of $Q_{\ell}{ }^{N}$. It follows that $\Gamma$ is Zariski dense in $Q_{\ell}^{N}$ if and only if $r=N$. This proves the result when $U$ is Abelian. 
Now suppose that $U$ is not Abelian. Note that the commutator subgroup $[\Gamma, \Gamma]$ is Zariski dense in $[U, U]$; it is clear that the Zariski closure of $[\Gamma, \Gamma]$ is contained in $[U, U]$. The reverse conclusion follows as the image of $\Gamma$ in the $Q_{\ell}$-rational points of the quotient of $U$ by the closure of $[\Gamma, \Gamma]$ is dense and Abelian, which implies that

$[U, U] \subseteq$ the Zariski closure of $[\Gamma, \Gamma]$ in $U$.

If $U$ is not Abelian, then there is a least $n$ for which $U \neq W_{-n} U$. Since the filtration $W$. is central, $W_{-n} U \supseteq[U, U]$. Using the fact that $[\Gamma, \Gamma]$ is Zariski dense in $[U, U]$ and also the fact that the result holds for $U /[U, U]$, it is not hard to see that $W_{-n} \Gamma$ is Zariski dense in $W_{-n} U$. By induction, the result holds for

$$
W_{-n} \Gamma \hookrightarrow W_{-n} U\left(Q_{\ell}\right)
$$

from which the result for $\Gamma \rightarrow U\left(Q_{\ell}\right)$ follows as

$$
\mathrm{Gr}_{-m}^{W} U= \begin{cases}\mathrm{Gr}_{-m}^{W} U / W_{-n} U, & m<n, \\ \mathrm{Gr}_{-m}^{W} W_{-n} U, & m \geqslant n .\end{cases}
$$

\section{Galois Actions on Geometric Fundamental Groups}

Fix a prime number $\ell$. Suppose that $F$ is a number field and that $X$ is a geometrically connected variety defined over $F$. Set $\bar{X}=X \otimes_{F} \bar{F}$. Denote the absolute Galois group of $F$ by $G_{F}$. We have the outer Galois action $\phi_{\ell}: G_{F} \rightarrow$ Out $\pi_{1}(\bar{X}, x)^{(\ell)}$ of $G_{F}$ on the pro- $\ell$ completion of the geometric fundamental group of $(X, x)$.

For a finite set of primes $S$ of the ring of integers $\mathcal{O}_{F}$, we denote the ring of $S$-integers by $\mathcal{O}_{F, S}$, and $\pi_{1}\left(\operatorname{Spec} \mathcal{O}_{F, S}, \operatorname{Spec} \bar{F}\right)$ by $G_{F, S}$. It is known that there is a finite set of primes $S$ such that $\phi_{\ell}$ factors through $G_{F} \rightarrow G_{F, S}$, so we have

$$
\phi_{S}: G_{F, S} \rightarrow \operatorname{Out} \pi_{1}(\bar{X}, x)^{(\ell)} .
$$

This $S$ can be taken as the union of the bad-reduction primes of $X$ and the primes above $\ell$, (cf. [9, Chapter XIII]). If $X=\mathbb{P}^{1}-\{0,1, \infty\}$ over $F=Q$, then we can take $S=\{\ell\}$.

\subsection{THE I-FILTRATION}

Define a filtration

$$
\text { Out } \pi_{1}(\bar{X}, x)^{(\ell)}=L^{0} \text { Out } \pi_{1}(\bar{X}, x)^{(\ell)} \supseteq L^{1} \text { Out } \pi_{1}(\bar{X}, x)^{(\ell)} \supseteq \cdots
$$

of the outer automorphism group of $\pi_{1}(\bar{X}, x)^{(\ell)}$ by

$$
\begin{aligned}
& L^{m} \text { Out } \pi_{1}(\bar{X}, x)^{(\ell)} \\
& \quad=\operatorname{ker}\left\{\text { Out } \pi_{1}(\bar{X}, x)^{(\ell)} \rightarrow \operatorname{Out}\left(\pi_{1}(\bar{X}, x)^{(\ell)} / L^{m+1} \pi_{1}(\bar{X}, x)^{(\ell)}\right)\right\}
\end{aligned}
$$


where

$$
\pi_{1}(\bar{X}, x)^{(\ell)}=L^{1} \pi_{1}(\bar{X}, x)^{(\ell)} \supseteq L^{2} \pi_{1}(\bar{X}, x)^{(\ell)} \supseteq \cdots
$$

denotes the lower central series of $\pi_{1}(\bar{X}, x)^{(\ell)}$. The $I$-filtration

$$
G_{F}=I_{\ell}^{0} G_{F} \supseteq I_{\ell}^{1} G_{F} \supseteq I_{\ell}^{2} G_{F} \supseteq \cdots
$$

of $G_{F}$ is the pull back of the filtration $L^{\bullet}$ of Out $\pi_{1}(\bar{X}, x)^{(\ell)}$ along the outer action:

$$
I_{\ell}^{m} G_{F}=\phi_{\ell}^{-1} L^{m} \text { Out } \pi_{1}(\bar{X}, x)^{(\ell)}=\operatorname{ker}\left\{G_{F} \rightarrow \text { Out }\left(\pi_{1}(\bar{X}, x)^{(\ell)} / L^{m+1}\right)\right\} .
$$

We can also pullback the filtration $L^{\bullet}$ of Out $\pi_{1}(\bar{X}, x)^{(\ell)}$ to define the $I$-filtration of $G_{F, S}$ :

$$
G_{F, S}=I_{\ell}^{0} G_{F, S} \supseteq I_{\ell}^{1} G_{F, S} \supseteq I_{\ell}^{2} G_{F, S} \supseteq \cdots
$$

By an elementary argument, the quotient mapping $G_{F} \rightarrow G_{F, S}$ induces isomorphisms

$$
\mathrm{Gr}_{I_{\ell}}^{n} G_{F, S} \cong \mathrm{Gr}_{I_{\ell}}^{n} G_{F} .
$$

Since $\left[I_{\ell}^{m} G_{F}, I_{\ell}{ }^{n} G_{F}\right] \subseteq I_{\ell}^{m+n} G_{F}$, the associated graded groups $\mathrm{Gr}_{I_{\ell}}^{>0} G_{F} \cong \mathrm{Gr}_{I_{\ell}}^{>0} G_{F, S}$ are Lie algebras over $Z_{\ell}$; the bracket is induced by the group commutator. (See [30], for example.)

\subsection{GALOIS ACTIONS ON UNIPOTENT COMPLETIONS}

Set $\mathcal{P}=\pi_{1}(\bar{X}, x)_{/ Q_{\ell}}^{\text {un }}$, the $\ell$-adic unipotent completion (see Section A.2) of $\pi_{1}(\bar{X}, x)$.

Remark 8.1. It follows from Theorem A.6 that for each imbedding $\sigma: F \hookrightarrow$ C, there is an isomorphism

$$
\mathcal{P}=\pi_{1}\left(X_{\sigma}, x\right)_{/ \mathrm{Q}}^{\mathrm{un}} \otimes_{\mathrm{Q}} Q_{\ell},
$$

where $X_{\sigma}$ denotes the complex variety obtained from $X$ via $\sigma$.

There is a proalgebraic group $\mathcal{O}$ ut $\mathcal{P}$ whose $K$-rational points form the group of continuous outer automorphisms of $\mathcal{P}(K)$ for each field extension $K$ of $Q_{\ell}$. (See Paragraph A.4.) By the functoriality of unipotent completion, the outer action of $G_{F, S}$ on $\pi_{1}(\bar{X}, x)$ induces a homomorphism $\Phi_{S}: G_{F, S} \rightarrow \mathcal{O}$ ut $\mathcal{P}$. As above, the lower central series

$$
\mathcal{P}=L^{1} \mathcal{P} \supseteq L^{2} \mathcal{P} \supseteq L^{3} \mathcal{P} \supseteq \cdots
$$

of $\mathcal{P}$ induces a filtration

$$
L^{0} \text { Out } \mathcal{P} \supseteq L^{1} \text { Out } \mathcal{P} \supseteq L^{2} \mathcal{O} \text { ut } \mathcal{P} \supseteq \cdots
$$


of $\mathcal{O}$ ut $\mathcal{P}$. Pulling this back along $\Phi_{S}$, we obtain a filtration

$$
G_{F, S}=\mathcal{I}_{\ell}^{0} G_{F, S} \supseteq \mathcal{I}_{\ell}^{1} G_{F, S} \supseteq \mathcal{I}_{\ell}^{2} G_{F, S} \supseteq \cdots
$$

of $G_{F, S}$.

PROPOSITION 8.2. For all $m \geqslant 0, I_{\ell}{ }^{m} G_{F, S} \subseteq \mathcal{I}_{\ell}{ }^{m} G_{F, S}$ and the natural map

$$
\mathrm{Gr}_{I_{\ell}}^{m} G_{F, S} \rightarrow \mathrm{Gr}_{\mathcal{I}_{\ell}}^{m} G_{F, S}
$$

has finite kernel and cokernel. In particular, if $m>0$, then

$$
\left(\mathrm{Gr}_{I_{\ell}}^{m} G_{F, S}\right) \otimes_{\mathrm{Z}_{\ell}} Q_{\ell} \cong\left(\mathrm{Gr}_{\mathcal{I}_{\ell}}^{m} G_{F, S}\right) \otimes_{\mathrm{Z}_{\ell}} Q_{\ell} .
$$

Proof. We will use Theorem A.3 and its notation. Set $\Gamma=\pi_{1}(\bar{X}, x)^{(\ell)}$. Since $\Gamma$ is a finitely generated pro- $\ell$ group, $L^{m} \Gamma \subseteq D^{m} \Gamma$ and $D^{m} \Gamma / L^{m} \Gamma$ is a finite $\ell$-group. Also, $L^{m} \Gamma=D^{m} \Gamma$ for all $m$ if and only if $\Gamma / L^{m} \Gamma$ is torsion free for all $m$.

Using universal mapping properties, one can show that the inclusion $\Gamma / D^{m} \Gamma \hookrightarrow\left(\mathcal{P} / L^{m} \mathcal{P}\right)\left(Q_{\ell}\right)$ is the $\ell$-adic unipotent completion of $\Gamma / D^{m} \Gamma$. It follows that the natural homomorphism Aut $\Gamma \rightarrow$ Aut $\mathcal{P}$ descends to a natural homomorphism

$$
\operatorname{Aut}\left(\Gamma / D^{m} \Gamma\right) \hookrightarrow \operatorname{Aut}\left(\mathcal{P} / L^{m} \mathcal{P}\right)
$$

which is injective. This induces a homomorphism

$$
\operatorname{Out}\left(\Gamma / D^{m} \Gamma\right) \rightarrow \operatorname{Out}\left(\mathcal{P} / L^{m} \mathcal{P}\right) \text {. }
$$

The kernel of this morphism is finite. This is equivalent to the assertion that $\operatorname{Inn}\left(\Gamma / D^{m} \Gamma\right)$ has finite index in $\operatorname{Aut}\left(\Gamma / D^{m} \Gamma\right) \cap \operatorname{Inn}\left(\mathcal{P} / L^{m} \mathcal{P}\right)$, which follows from the compactness of $\operatorname{Aut}\left(\Gamma / D^{m} \Gamma\right)$ ( $\Gamma$ is finitely generated as a pro- $\ell$ group $)$ and the openness of $\Gamma / D^{m} \Gamma$ in $\mathcal{P} / L^{m} \mathcal{P}\left(\mathrm{Q}_{\ell}\right)$, a consequence of Lemma 7.5. Since $D^{m} \Gamma / L^{m} \Gamma$ is a finite $\ell$-group, it follows that the kernel of both

$$
\operatorname{Out}\left(\Gamma / L^{m} \Gamma\right) \rightarrow \operatorname{Out}\left(\Gamma / D^{m} \Gamma\right) \quad \text { and } \operatorname{Out}\left(\Gamma / L^{m} \Gamma\right) \rightarrow \operatorname{Out}\left(\mathcal{P} / L^{m} \mathcal{P}\right)
$$

are finite from which it follows that $I_{\ell}{ }^{m} G_{F, S}$ is a finite index subgroup of $\mathcal{I}_{\ell}{ }^{m} G_{F, S}$.

Because the sequence

$$
1 \rightarrow\left(I_{\ell}{ }^{m} \cap \mathcal{I}_{\ell}^{m+1}\right) / I_{\ell}^{m+1} \rightarrow I_{\ell}^{m / I} \ell^{m+1} \stackrel{\phi}{\rightarrow} \mathcal{I}_{\ell}^{m} / \mathcal{I}_{\ell}^{m+1} \rightarrow \mathcal{I}_{\ell}^{m} /\left(I_{\ell}^{m}+\mathcal{I}_{\ell}^{m+1}\right) \rightarrow 1
$$

is exact, the kernel and cokernel of $\phi$ are finite.

Since $\left[I_{\ell}{ }^{m}, I_{\ell}{ }^{n}\right] \subseteq I_{\ell}{ }^{m+n}$ and $\left[\mathcal{I}_{\ell}{ }^{m}, \mathcal{I}_{\ell}{ }^{n}\right] \subseteq \mathcal{I}_{\ell}{ }^{m+n}, \mathrm{Gr}_{I_{\ell}}^{m} G_{F, S}$ and $\mathrm{Gr}_{\mathcal{I}_{\ell}}^{m} G_{F, S}$ are abelian pro- $\ell$ groups whenever $m>0$. Thus it follows that the kernel and cokernel of the mapping on each associated graded is a finite abelian $\ell$-group when $m>0$.

\subsection{THE GALOIS IMAGE}

Choose an $F$-rational base point - either a geometric point $x$ or a tangential base point anchored at an $F$-rational point of a smooth completion of $X$. Denote the Lie algebra of $\mathcal{P}$ by $\mathfrak{p}$. It is well known that $H_{\mathrm{et}}^{1}\left(\bar{X}, Q_{\ell}\right)$ is isomorphic to $\operatorname{Hom}^{\text {cts }}\left(\pi_{1}(\bar{X}, x), Q_{\ell}\right)$ as a continuous $G_{F, S}$-module. 
Denote the Zariski closure of the image of $G_{F, S}$ in $\mathcal{A}$ ut $\mathcal{P}$ by $\widehat{\mathcal{G}}_{F, S}$ and its prounipotent radical by $\widehat{\mathcal{U}}_{F, S}$. Denote the Zariski closure of the image of $G_{F, S}$ in $\mathcal{O}$ ut $\mathcal{P}$ by $\mathcal{G}_{F, S}$ and its prounipotent radical by $\mathcal{U}_{F, S}$. There is a natural homomorphism $\widehat{\mathcal{G}}_{F, S} \rightarrow \mathcal{G}_{F, S}$.

PROPOSITION 8.3. If $H_{e t}^{1}\left(\bar{X}, Q_{\ell}(1)\right)$ is a trivial $G_{F, S}$-module, then

(i) the natural homomorphism $G_{F, S} \rightarrow \mathcal{A u t} \mathcal{P} \rightarrow$ Aut $H_{1}(\mathfrak{p})$ is the composition of the $\ell$-adic cyclotomic character with the inclusion of the scalar matrices;

(ii) the subgroup $\widehat{\mathcal{G}}_{F, S}$ of $\mathcal{A}$ ut $\mathcal{P}$ is a negatively weighted extension of the scalar matrices $\mathbb{G}_{m}$ by $\widehat{\mathcal{U}}_{F, S}$ with respect to the central cocharacter w: $x \mapsto x^{-2}$;

(iii) the subgroup $\mathcal{G}_{F, S}$ of $\mathcal{O}$ ut $\mathcal{P}$ is a negatively weighted extension of the scalar matrices $\mathrm{G}_{m}$ by $\mathcal{U}_{F, S}$ with respect to the central cocharacter $w: x \mapsto x^{-2}$;

(iv) The homomorphism $G_{F, S} \rightarrow$ Aut $\mathcal{U}\left(\mathrm{Q}_{\ell}\right)$ induces surjective homomorphisms

$\mathcal{A}_{F, S} \rightarrow \widehat{\mathcal{G}}_{F, S} \rightarrow \mathcal{G}_{F, S}$

(v) $\mathfrak{p}$ has a natural weight filtration which is essentially its lower central series:

$W_{-2 m} \mathfrak{p}=L^{m} \mathfrak{p} \quad$ and $\quad \mathrm{Gr}_{2 m+1}^{W} \mathfrak{p}=0$ for all $m ;$

(vi) the weight filtrations on $\mathfrak{p}$, Der $\mathfrak{p}$ and OutDer $\mathfrak{p}$ are related by

$\operatorname{Gr}_{n}^{W} \operatorname{OutDer} \mathfrak{p}=\left(\mathrm{Gr}_{n}^{W} \operatorname{Der} \mathfrak{p}\right) /\left(\mathrm{Gr}_{n}^{W} \mathfrak{p}\right)$ and $\operatorname{Gr}_{\bullet}^{W} \operatorname{Der} \mathfrak{p} \cong \operatorname{DerGr}_{\bullet}^{W} \mathfrak{p} ;$

(vii) the weight filtration of $\widehat{\mathcal{U}}_{K, T}$ is characterized by

$W_{-2 m} \widehat{\mathcal{U}}_{F, S}=\operatorname{ker}\left\{\widehat{\mathcal{U}}_{F, S} \rightarrow \mathcal{A u t}\left(\mathcal{P} / L^{m+1} \mathcal{P}\right)\right\}$ and $\operatorname{Gr}_{2 m+1}^{W} \widehat{\mathcal{U}}_{F, S}=1$

for all $m$;

(viii) the weight filtration of $\mathcal{U}_{F, S}$ is characterized by

$W_{-2 m} \mathcal{U}_{F, S}=\operatorname{ker}\left\{\mathcal{U}_{F, S} \rightarrow \mathcal{O u t}\left(\mathcal{P} / L^{m+1} \mathcal{P}\right)\right\} \quad$ and $\operatorname{Gr}_{2 m+1}^{W} \mathcal{U}_{F, S}=1$

for all $m$.

Proof. The first assertion follows from the assumption using the natural isomorphism $H_{1}(\bar{X}) \cong H_{1}(\mathfrak{p})$. Since the bracket

$$
\mathrm{Gr}_{L}^{1} \mathfrak{p} \otimes \mathrm{Gr}_{L}^{n} \mathfrak{p} \rightarrow \mathrm{Gr}_{L}^{n+1} \mathfrak{p}
$$

is surjective and $G_{F, S}$ equivariant, it follows that $G_{F, S}$ acts on $\operatorname{Gr}_{L}^{m} \mathfrak{p}$ via the $m$ th power of the cyclotomic character. This implies the second and third assertions as it implies that every derivation of $\mathfrak{p}$ that acts trivially on $\mathrm{Gr}_{L}^{\bullet} \mathfrak{p}$ has negative weight. The fourth follows from the universality of $\mathcal{A}_{F, S}$. Because $\mathfrak{p}$ is a module over $\mathcal{A}_{F, S}$, it has a natural weight filtration. Since $G_{F, S}$ acts on $\operatorname{Gr}_{L}^{n} \mathfrak{p}$ via the $n$th power of the cyclotomic character, next assertion follows. The remaining assertions follow as Der $\mathfrak{p}$ and OutDer $\mathfrak{p}$ are negatively weighted $G_{F, S}$-modules. 
THEOREM 8.4. If $H_{\mathrm{et}}^{1}\left(\bar{X}, Q_{\ell}(1)\right)$ is a trivial $G_{F, S}$-module, then there is a graded Lie algebra surjection $\mathrm{Gr}_{\bullet}^{W_{\mathfrak{f}}} F, \mathrm{Gr}_{\bullet}^{W} \mathfrak{u t}_{F, S}$ and an isomorphism

$$
\mathrm{Gr}_{\bullet}^{W} \mathfrak{u}_{F, S} \cong\left(\mathrm{Gr}_{I_{\ell}}^{>0} G_{F, S}\right) \otimes_{Z_{\ell}} Q_{\ell} .
$$

Proof. The surjection $\mathcal{A}_{F, S} \rightarrow \mathcal{G}_{F, S}$ restricts to a surjection $\mathcal{K}_{F, S} \rightarrow \mathcal{U}_{F, S}$, which implies that $I_{\ell}{ }^{1} G_{F, S} \rightarrow \mathcal{U}_{F, S}\left(Q_{\ell}\right)$ has Zariski dense image. By strictness, it induces a surjection $\mathrm{Gr}_{\bullet}^{W} \mathfrak{f}_{F, S} \rightarrow \mathrm{Gr}_{\bullet}^{W} \mathfrak{t a t}_{F, S}$. From Proposition 8.3, it follows that $\mathcal{I}_{\ell}{ }^{m} G_{F, S}$ is the inverse image of $W_{-2 m} \mathcal{G}_{F, S}$ under the natural mapping $G_{F, S} \rightarrow \mathcal{G}_{F, S}$. There is thus an injection

$$
\mathrm{Gr}_{\mathcal{I}_{\ell}}^{m} G_{F, S} \hookrightarrow \mathrm{Gr}_{-2 m}^{W} \mathcal{G}_{F, S} .
$$

Proposition 8.2, Proposition 7.1 and Lemma 7.5 imply that this induces isomorphisms

$$
\left(\mathrm{Gr}_{I_{\ell}}^{m} G_{F, S}\right) \otimes_{Z_{\ell}} Q_{\ell} \cong\left(\mathrm{Gr}_{\mathcal{I}_{\ell}}^{m} G_{F, S}\right) \otimes_{Z_{\ell}} Q_{\ell} \cong \operatorname{Gr}_{-2 m}^{W} \mathcal{U}_{F, S} \cong \operatorname{Gr}_{-2 m}^{W} \mathfrak{u}_{F, S} .
$$

whenever $m>0$.

\subsection{PROOF OF CONJECTURE 1}

Since $\mathbb{P}^{1}-\{0,1, \infty\}$ has everywhere good reduction, the Galois representation

$$
\phi_{\ell}: G_{Q} \rightarrow \text { Out } \pi_{1}(\mathrm{P}(1 / \mathrm{Q})-\{0,1, \infty\})^{(\ell)}
$$

factors through the projection $G_{Q} \rightarrow G_{\ell}=G_{Q,\{\ell\}}[15$, Thm. 1]. It is standard that $H_{\mathrm{et}}^{1}\left(\mathrm{P}(1 / \mathrm{Q})-\{0,1, \infty\}, \mathrm{Q}_{\ell}(1)\right)$ is a trivial $G_{\ell}$-module. Now apply Theorem 8.4 with $X=\mathbb{P}^{1}-\{0,1, \infty\}, F=\mathbb{Q}, S=\{\ell\}, \mathfrak{f}_{F, S}=\mathfrak{f}_{\ell}$. Theorem 7.3 says that $\mathrm{Gr}_{\bullet}^{W_{\ell}}$ is generated by elements

$$
\sigma_{2 n+1} \in \mathrm{Gr}_{-2(2 n+1)^{W}}^{\mathfrak{f}_{\ell}} \quad n \geqslant 0 .
$$

Conjecture 1 now follows from Theorem 8.4 and the following lemma, which implies that the image of $\sigma_{1}$ in

$$
\left(\mathrm{Gr}_{I}^{>0} G_{\ell}\right) \otimes \mathrm{Q}_{\ell} \cong \mathrm{Gr}_{\bullet}^{W} \mathfrak{t}_{\mathrm{Q},\{\ell\}}
$$

is trivial.

LEMMA 8.5. We have $\mathrm{Gr}_{-2}^{W}$ OutDer $\mathfrak{p}=0$, so that $\mathrm{Gr}_{-2}^{W} \mathfrak{l}_{\mathrm{Q},\{\ell\}}=0$.

Proof. Since $\mathrm{Gr}_{\mathfrak{p}}^{W} \mathfrak{p}$ is isomorphic to the free Lie algebra generated by $H_{1}(\mathfrak{p})$, it follows that $\mathrm{Gr}_{-4}^{W} \mathfrak{p} \cong Q_{\ell}(2)$. By Proposition 8.3 we have

$$
\mathrm{Gr}_{-2}^{W} \operatorname{Der} \mathfrak{p} \cong \mathrm{Gr}_{-2}^{W} \operatorname{Der} \mathrm{Gr}_{\bullet}^{W} \mathfrak{p} \cong \operatorname{Hom}\left(H_{1}(\mathfrak{p}), \mathrm{Gr}_{-4}^{W} \mathfrak{p}\right) \cong H_{1}(\mathfrak{p})
$$

The non-degeneracy of the Lie bracket

$$
H_{1}(\mathfrak{p}) \otimes H_{1}(\mathfrak{p}) \rightarrow \mathrm{Gr}_{-4}^{W} \mathfrak{p} \cong \mathrm{Q}_{\ell}(2)
$$

implies that the mapping $H_{1}(\mathfrak{p}) \rightarrow \operatorname{Hom}\left(H_{1}(\mathfrak{p}), \mathrm{Gr}_{-4}^{W} \mathfrak{p}\right)$, given by taking inner deri- 
vations, is an isomorphism. Thus

$$
\mathrm{Gr}_{-2}^{W} \text { OutDer } \mathfrak{p} \cong \mathrm{Gr}_{-2}^{W} \operatorname{Der} \mathfrak{p} / \mathrm{Gr}_{-2}^{W} \mathfrak{p} \cong \operatorname{Hom}\left(H_{1}(\mathfrak{p}), \mathrm{Gr}_{-4}^{W} \mathfrak{p}\right) / H_{1}(\mathfrak{p})=0 .
$$

Remark 8.6. Our proof says nothing about the nontriviality of the

$$
\sigma_{2 m+1} \in\left(\mathrm{Gr}_{I_{\ell}}^{\bullet} G_{\ell}\right) \otimes Q_{\ell}
$$

Ihara's work [16] implies that when $m \geqslant 1$, all are nontrivial in $H_{1}\left(\left(\mathrm{Gr}_{I_{\ell}}^{\bullet} G_{\ell}\right) \otimes Q_{\ell}\right)$. Combined with Theorem 7.3, this implies that

$$
H_{1}\left(\left(\mathrm{Gr}_{I_{\ell}}^{\bullet} G_{\ell}\right) \otimes Q_{\ell}\right)=\bigoplus_{m \geqslant 1} Q_{\ell}(2 m+1)
$$

where the copy of $Q_{\ell}(2 m+1)$ is spanned by $\sigma_{2 m+1}$. Ihara [16] also uses power series methods to establish nonvanishing results for some brackets of the $\sigma_{j}$. Improvements can be found in [22] and [36].

Ihara defined a certain Z-lie algebra $\mathcal{D}$ called the stable derivation algebra, which is a subalgebra of outer derivations of free Z-Lie algebra with two generators. It is proved that $\mathrm{Gr}_{I}^{>0} G_{\ell}$ is contained in $\mathcal{D} \otimes Z_{\ell}$ for every prime $\ell$. He considered the problem of whether $\mathcal{D}$ is generated by certain derivations $D_{2 m+1}$, which are analogues of the $\sigma_{2 m+1}$. He found a mysterious congruence [17]

$$
2\left[D_{3}, D_{9}\right]-27\left[D_{5}, D_{7}\right] \equiv 0 \bmod 691
$$

when $\ell=691$. This suggests that one might have

$$
2\left[\sigma_{3}, \sigma_{9}\right]-27\left[\sigma_{5}, \sigma_{7}\right] \equiv 0 \bmod 691
$$

in $\mathrm{Gr}_{I_{\ell}}^{>0} G_{\ell}$ for $\ell=691$, hence this Lie algebra might not be generated by the elements $\sigma_{i}$ over $Z_{\ell}$ for $\ell=691$. This is not currently known, but Sharifi announced interesting progress: for $\ell$ regular, the freeness of $\mathrm{Gr}_{I_{\ell}}^{>0} G_{\ell} \otimes Q_{\ell}$ implies that $\mathrm{Gr}_{I_{\ell}}^{>0}$ itself is generated by the elements $\sigma_{i}$, and for $\ell$ irregular, a conjecture of Greenberg's implies that $\mathrm{Gr}_{I_{\ell}}^{>0} G_{\ell}$ is not free on $\sigma_{i}$.

\subsection{GONCHAROV'S CONJECTURE}

Goncharov [11] considers the varieties

$$
X_{N}:=\mathbb{P}^{1}-\left\{0, \mu_{N}, \infty\right\}
$$

where $\mu_{N}$ denotes the group of $N$ th roots of unity. Take $F$ to be $\mathrm{Q}\left(\mu_{N}\right)$ and $S$ to be the set of primes in $\mathcal{O}_{F}$ that lie over $N \ell$. Let $v=\overrightarrow{01}$, the tangent vector at 0 that points towards 1. The Galois representation

$$
\hat{\phi}_{\ell}: G_{F} \rightarrow \operatorname{Aut} \pi_{1}(\bar{X}, v)^{(\ell)}
$$

is unramified outside $S$ by the Smooth Base Change Theorem [9, Chapt. XIII], so it factors through a representation

$$
\hat{\phi}_{S}: G_{F, S} \rightarrow \operatorname{Aut} \pi_{1}(\bar{X}, v)^{(\ell)} .
$$


Denote the $\ell$-adic unipotent completion of $\pi_{1}(\bar{X}, v)^{(\ell)}$ by $\mathcal{P}$. By functoriality, $\hat{\phi}_{\ell}$ and $\hat{\phi}_{S}$ induce homomorphisms $G_{F} \rightarrow G_{F, S} \rightarrow \operatorname{Aut} \mathcal{P}\left(Q_{\ell}\right)$. By Proposition 8.3, this induces a homomorphism

$$
\hat{\Phi}_{S}: \mathcal{A}_{F, S} \rightarrow \mathcal{A u t} \mathcal{P} .
$$

Goncharov considers the Zariski closure of the image of

$$
G_{\mathrm{Q}\left(\mu_{\ell} \infty\right)}:=\operatorname{Gal}\left(\overline{\mathrm{Q}} / \mathrm{Q}\left(\mu_{\ell^{\infty}}\right)\right)
$$

in $\mathcal{A}$ ut $\mathcal{P}$. Since

$$
G_{\mathrm{Q}\left(\mu_{\ell} \infty\right)}=\operatorname{ker}\left\{\chi_{\ell}: G_{F} \rightarrow Z_{\ell}^{\times}\right\},
$$

Theorem 7.1 implies that this is the image $\widehat{\mathcal{U}}_{F, S}$ of $\mathcal{K}_{F, S}$ in $\mathcal{A u t} \mathcal{P}$.

THEOREM 8.7. For all $N \geqslant 1$, the Lie algebra of the Zariski closure of the image of $G_{\mathrm{Q}\left(\mu_{e}\right)} \rightarrow$ Aut $\mathcal{P}$ is a quotient of $\mathfrak{f}_{F, S}$, and is therefore generated topologically by a lift of

$$
\bigoplus_{n \geqslant 0} H_{\mathrm{et}}^{1}\left(\operatorname{Spec} \mathcal{O}_{\mathrm{Q}\left(\mu_{N}\right), S}, Q_{\ell}(n)\right)^{*} \otimes Q_{\ell}(n) .
$$

When $N=1$, this proves the generation portion of Conjecture 2.1 in [11].

\section{9. $\ell$-Adic Mixed Tate Modules Over Spec $\mathcal{O}_{F, S}$}

In this section, we show how weighted completion of Galois groups can be used to prove $\ell$-adic versions of Deligne's Conjectures $[5, \S 8]$ on mixed Tate motives over the spectrum of the ring of $S$-integers in a number field. Such $\ell$-adic versions of these conjectures have previously been proved by Beilinson and Deligne by different, but equivalent, methods in their unpublished manuscript [1].

\subsection{DELIGNE'S CONJECTURES}

Suppose that $F$ is a number field and that $S$ is a finite subset of Spec $\mathcal{O}_{F}$. Deligne $[5,8.2]$ conjectures that there is a category of mixed Tate motives over Spec $\mathcal{O}_{F, S}$ and that in this category, $\operatorname{Ext}^{1}(\mathrm{Q}(0), \mathrm{Q}(n))=K_{2 n-1}\left(\mathcal{O}_{F, S}\right) \otimes \mathrm{Q}$. The tannakian fundamental group of this category is of the form $\mathrm{G}_{m} \times U$ where $U$ is prounipotent. He further conjectures $[5,8.9 .5]$ that the weight graded Lie algebra of $U$ is freely generated by

$$
\bigoplus_{n \geqslant 1} K_{2 n-1}\left(\mathcal{O}_{F, S}\right) \otimes \mathrm{Q} \text {. }
$$

(The generation statement is equivalent to the first conjecture above.)

The analogue of this conjecture for mixed Tate motives over a number field has been solved by Goncarov [10], who used results of Voevodsky [37] and Levine [20] 
to construct the category of mixed Tate motives over a number field. He has also made a working definition of mixed Tate motives over the ring of $S$-integers in a number field.

Here we formulate and prove an $\ell$-adic version of this. Fix a rational prime $\ell$. Beilinson and Deligne had given a similar construction in the unpublished manuscript [1].

\subsection{THE CASE $\{\ell\}$ CONTAINED IN $S$}

First, we shall assume that $S$ contains all primes that lie over $\ell$. Later we shall remove this hypothesis. In this case, it is natural to define the category of $\ell$-adic mixed Tate modules over Spec $\mathcal{O}_{F, S}$ to be the category of weighted $G_{F, S}$-modules (see Definition 4.2). Denote this category by $\mathcal{M T M}_{\ell}\left(\mathcal{O}_{F, S}\right)$.

LEMMA 9.1. The category $\mathcal{M T M}_{\ell}\left(\mathcal{O}_{F, S}\right)$ is equivalent to the category of finitedimensional representations of $\mathcal{A}_{F, S}$. For each $n \in Z$ and $i \geqslant 0$, there is a natural isomorphism

$$
\operatorname{Ext}_{\left.\mathcal{M T M} \mathcal{T}_{\ell, S}\right)}^{i}\left(\mathrm{Q}_{\ell}, \mathrm{Q}_{\ell}(n)\right) \cong H^{i}\left(\mathcal{A}_{F, S}, \mathrm{Q}_{\ell}(n)\right) .
$$

Proof. This follows directly from [14] - see Remark 4.3.

If $\mathcal{U}$ is prounipotent with Lie algebra $\mathfrak{k}$, then $H^{\bullet}(\mathcal{U}) \cong H_{\text {cts }}^{\bullet}(\mathfrak{l})$, since the category of $\mathcal{U}$-modules is equivalent to the category of continuous $\mathfrak{t}$-modules.

THEOREM 9.2. There are natural isomorphisms

$$
\operatorname{Ext}_{\mathcal{M} \operatorname{TM}_{\ell}\left(\mathcal{O}_{F, S}\right)}^{i}\left(Q_{\ell}, Q_{\ell}(n)\right) \cong \begin{cases}Q_{\ell}, & \text { when } i=n=0, \\ H_{\mathrm{cts}}^{1}\left(G_{F, S}, Q_{\ell}(n)\right), & \text { when } i=1 \text { and } n>0, \\ 0, & \text { otherwise. }\end{cases}
$$

Consequently, for all $n \in Z_{>0}$, there is a natural isomorphism

$$
\operatorname{Ext}_{\mathcal{M T M}}^{1}\left(_{\left.\mathcal{O}_{F, S}\right)}\left(\mathrm{Q}_{\ell}, \mathrm{Q}_{\ell}(n)\right) \cong K_{2 n-1}\left(\operatorname{Spec} \mathcal{O}_{F, S}\right) \otimes \mathrm{Q}_{\ell}\right.
$$

Proof. Because of the previous lemma, we only need compute $H^{i}\left(\mathcal{A}_{F, S}, Q_{\ell}(n)\right)$. It is proved in [19] that there is a Leray-Serre type spectral sequence

$$
H^{s}\left(\mathrm{G}_{m}, H^{t}\left(\mathcal{K}_{F, S}, \mathrm{Q}_{\ell}(n)\right)\right) \Rightarrow H^{s+t}\left(\mathcal{A}_{F, S}, \mathrm{Q}_{\ell}(n)\right) .
$$

Since $\mathrm{G}_{m}$ is reductive and $H^{\bullet}\left(\mathcal{K}_{F, S}\right) \cong H_{\text {cts }}^{\bullet}\left(\mathfrak{f}_{F, S}\right)$, it follows that

$$
H^{i}\left(\mathcal{A}_{F, S}, Q_{\ell}(n)\right)=\left[H_{\mathrm{cts}}^{i}\left(\mathfrak{f}_{F, S}\right) \otimes Q_{\ell}(n)\right]^{\mathrm{G}_{m}} .
$$

Since $\mathfrak{f}_{F, S}$ is negatively weighted, the right-hand side is zero when $n \leqslant 0$. Since $\mathrm{Gr}_{\bullet}^{W} \mathfrak{f}_{F, S}$ is free and $\mathrm{Gr}_{\bullet}^{W}$ is exact,

$$
\mathrm{Gr}_{\bullet}^{W} H_{\mathrm{cts}}^{i}\left(\mathfrak{f}_{F, S}\right)=H^{i}\left(\mathrm{Gr}_{\bullet}^{W} \mathfrak{f}_{F, S}\right)=0
$$

when $i>1$ and $H_{\mathrm{cts}}^{i}\left(\mathfrak{f}_{F, S}\right)$ vanishes if $i \geqslant 2$. When $i=1$, Theorem 7.2 gives the result. For $i=0$, the assertion is obvious. 
The last assertion follows from Proposition B.2 and the fact that the regulator mappings

$$
c_{1}: K_{2 n-1}\left(\mathcal{O}_{F, S}\right) \otimes Q_{\ell} \rightarrow H_{\mathrm{et}}^{1}\left(\operatorname{Spec} \mathcal{O}_{F, S}, Q_{\ell}(n)\right)
$$

are isomorphisms for all $n$ and $\ell$. This is due to Soule [33] when $\ell \neq 2$ and Rognes and Weibel [27] when $\ell=2$.

\subsection{CONSTRAINED WEIGHTED COMPLETION}

In order to handle the case where $\ell$ is not contained in $S$, we need to consider a variant of weighted completion. We use the notation of Section 4. Suppose that $P$ is a property of weighted $\Gamma$-modules that is closed under direct sums, tensor products, taking duals, and taking subquotients. Suppose also that the trivial representation $k$ has property $P$.

The category of finite-dimensional weighted $\Gamma$-modules with property $P$ is a full subcategory of the category of weighted $\Gamma$-modules. In fact, it is a neutral full subtannakian category of the category of weighted $\Gamma$-modules. The weighted completion of $\Gamma$ constrained by $P$ is defined to be the tannakian fundamental group $\mathcal{G}_{P}$ of this subcategory. Denote the kernel of $\mathcal{G}_{P} \rightarrow R$ by $\mathcal{U}_{P}$ and its Lie algebra by $\mathfrak{t}_{P}$.

By construction, the categories of weighted $\Gamma$-modules with property $P$ and the category of finite-dimensional $\mathcal{G}_{P}$-modules are equivalent. Suppose that $V$ is a weighted $\Gamma$-module with property $P$. Denote by $H_{\text {cts }}^{1}(\Gamma, V)_{P}$ the subgroup of $H_{\mathrm{cts}}^{1}(\Gamma, V)$ generated by the classes of extensions

$$
0 \rightarrow V \rightarrow E \rightarrow k \rightarrow 0
$$

of $\Gamma$-modules in which $E$ is also a weighted $\Gamma$-module with property $P$.

PROPOSITION 9.3. For all negatively weighted $\Gamma$-modules $V$ with property $P$, there is a natural isomorphism

$$
H^{1}\left(\mathcal{G}_{P}, V\right) \stackrel{\simeq}{\longrightarrow} H_{\mathrm{cts}}^{1}(\Gamma, V)_{P} .
$$

When $R$ is reductive and each $H_{\mathrm{cts}}^{1}\left(\Gamma, V_{\alpha}\right)$ is finite-dimensional,

$$
H^{1}\left(\mathcal{U}_{P}\right) \cong H_{\mathrm{cts}}^{1}\left(\mathfrak{u}_{P}\right) \cong \bigoplus_{\{\alpha: n(\alpha)<0\}} H_{\mathrm{cts}}^{1}\left(\Gamma, V_{\alpha}\right)_{P} \otimes V_{\alpha}^{*}
$$

Here we are using the notation of Paragraph 4.3.

Proof. The first statement follows because $H_{\text {cts }}^{1}(\Gamma, V)_{P}$ classifies extensions of $k$ by $V$ with property $P$. Since $V$ is negative, each extension of $k$ by $V$ is a weighted $\Gamma$-module and hence a $\mathcal{G}_{P}$-module. It therefore determines an element of $H^{1}\left(\mathcal{G}_{P}, V\right)$. 
The second assertion follows using the Leray-Serre Spectral Sequence [19] for the extension

$$
1 \rightarrow \mathcal{U}_{P} \rightarrow \mathcal{G}_{P} \rightarrow R \rightarrow 1
$$

and the fact that $R$ is reductive, which implies that $H^{s}\left(R, H^{t}\left(\mathcal{U}_{P}, V_{\alpha}\right)\right)$ vanishes whenever $s>0$. We also use the fact that $H_{\text {cts }}^{\bullet}\left(\mathfrak{u t}_{P}\right) \cong H^{\bullet}\left(\mathcal{U}_{P}\right)$.

To obtain the analogue of Theorem 4.9 for $H_{\mathrm{cts}}^{2}\left(\mathfrak{l t}_{P}\right)$, we need to impose an extra condition on $P$.

DEFINITION 9.4. Assume that there is a natural number $b$ such that for all weighted $\Gamma$-modules $V, \mathrm{Gr}_{m}^{W} V=0$ when $m$ is not a multiple of $b$. We say that property $P$ of weighted $\Gamma$-modules has the bootstrap property with index $b$ if $V$ has property $P$ whenever $W_{m} V / W_{m-2 b} V$ has property $P$ for all $m$.

Note that the assumption is always satisfied when $b=1$ and that it is satisfied when $b=2$ in the mixed Tate case.

THEOREM 9.5. Assume that $R$ is reductive and that $H_{\mathrm{cts}}^{2}\left(\Gamma, V_{\alpha}\right)$ is finite-dimensional for each irreducible representation $V_{\alpha}$ of $R$. Let $P$ be a property of weighted $\Gamma$-modules that it is closed under tensor products, direct sums, and taking duals and subquotients as $\Gamma$-modules. Suppose also that the trivial module $k$ has property $P$. If there is $a b \geqslant 1$ such that $P$ has the bootstrap property with index $b$, then there is $a$ natural injection

$$
H_{\mathrm{cts}}^{2}\left(\mathfrak{H}_{P}\right) \hookrightarrow \bigoplus_{\{\alpha: n(\alpha) \leqslant-2 n\}} H_{\mathrm{cts}}^{2}\left(\Gamma, V_{\alpha}\right) \otimes V_{\alpha}^{*},
$$

where $n$ is any positive integer such that $W_{n-1} H_{\mathrm{cts}}^{1}\left(\mathfrak{l}_{P}\right)=0$ or, equivalently, $W_{-n} \mathfrak{l}_{P}=\mathfrak{l}_{P}$.

Proof. As in the proof of Proposition 9.3, the Leray-Serre spectral sequence implies that $H^{2}\left(\mathcal{U}_{P}, V_{\alpha}\right) \cong\left[H_{\text {cts }}^{2}\left(\mathfrak{l t}_{P}\right) \otimes V_{\alpha}\right]^{R}$. It thus suffices to prove that the natural mapping

$$
H^{2}\left(\mathcal{G}_{P}, V_{\alpha}\right) \rightarrow H_{\text {cts }}^{2}\left(\Gamma, V_{\alpha}\right)
$$

is injective. Let $[\gamma]$ be a class in the left-hand side corresponding to a two-step extension

$$
1 \rightarrow V_{\alpha} \rightarrow E_{2} \rightarrow E_{1} \rightarrow k \rightarrow 1
$$

Since all $\mathcal{G}_{P}$-modules are locally finite, we may assume $E_{2}, E_{1}$ to be finite-dimensional. By exactness of the weight filtration and the reductivity of $R$, we may assume that $W_{0} E_{1}=E_{1}, W_{n(\alpha)} E_{1}=0$, and that $W_{-1} E_{2}=E_{2}, W_{n(\alpha)-1} E_{2}=0$. We assume that $[\gamma]$ is a trivial class in the right-hand side of (9). Since $\operatorname{Ext}_{\Gamma}^{2}\left(k, V_{\alpha}\right) \rightarrow H_{\mathrm{cts}}^{2}\left(\Gamma, V_{\alpha}\right)$ is 
injective (cf. Theorem A.1 in [14]), it follows that $[\gamma]$ is a trivial class as a two step extension of $\Gamma$-modules. Then [38, p. 575] implies the existence of a $\Gamma$-module $E$ with $0 \rightarrow V_{\alpha} \rightarrow E \rightarrow E_{1} \rightarrow 0$ and $0 \rightarrow E_{2} \rightarrow E \rightarrow k \rightarrow 0$. Now the bootstrap property says that $E$ has property $P$ and, hence, is a weighted $\Gamma$-module with property $P$. Thus this is a $\mathcal{G}_{P}$ module, which says that $[\gamma]$ is the trivial class as an extension of $\mathcal{G}_{P}$-modules, too. Thus injectivity is proved.

\subsection{THE CASE WHERE $\ell$ IS NOT CONTAINED IN $S$}

We now show how to remove the hypothesis that $S$ contain all primes over $\ell$. Let $[\ell]$ denote the set of primes of $F$ above the rational prime $\ell$. Assume that $S$ does not contain $[\ell]$. Then we define the category $\mathcal{M T M}_{\ell}\left(\mathcal{O}_{F, S}\right)$ of $\ell$-adic mixed Tate modules over Spec $\mathcal{O}_{F, S}$ to be the full subcategory of $\mathcal{M T M}_{\ell}\left(\mathcal{O}_{F, S \cup \ell \ell]}\right)$ consisting of the modules $M$ with the property that for every prime $\mathfrak{p} \in[\ell] \backslash S$, the representation $G_{F_{\mathfrak{p}}} \rightarrow$ $G_{F, S \cup[\ell]} \rightarrow$ Aut $M$ is crystalline, where $G_{F_{p}}$ is the decomposition group of $G_{F}$ at the prime $\mathfrak{p}$, and $F_{\mathfrak{p}}$ is the completion of $F$ at $\mathfrak{p}$. (See $[3,8]$ for crystalline representations.)

Let $P$ be the condition that a weighted $G_{F, S \cup[\ell]}$-module is crystalline at every prime

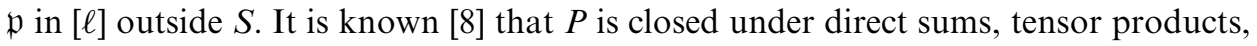
taking subquotients and duals, and that the trivial representation is crystalline. We can therefore consider the weighted completion of $G_{F, S \cup[\ell]}$ with respect to the cyclotomic character and constrained by $P$ - it is the Tannakian fundamental group of $\mathcal{M T M}_{\ell}\left(\mathcal{O}_{F, S}\right)$. We denote this completion by $\mathcal{A}_{F, S}$, its prounipotent radical by $\mathcal{K}_{F, S}$ and the Lie algebra of $\mathcal{K}_{F, S}$ by $\mathfrak{f}_{F, S}$.

The group $H_{\mathrm{cts}}^{1}\left(G_{F, S}, V\right)_{P}$ is the finite part $H_{\mathrm{cts} f}^{1}\left(G_{K}, V\right)$ of $H_{\mathrm{cts}}^{1}\left(G_{K}, V\right)$, which is defined in [3, (3.7.2)] and [3, p. 354] where it is denoted simply by $H_{f}^{1}\left(G_{K}, V\right)$.

One can show that the property $P$ has the bootstrap property for $b=2$. The key point of the proof is that for a short exact sequence

$$
0 \rightarrow V_{1} \rightarrow V_{2} \rightarrow V_{3} \rightarrow 0
$$

of crystalline $\ell$-adic representations of $G_{F_{p}}$, we have, by [3, Cor. 3.8.4], a long exact sequence

$$
\begin{aligned}
0 & \rightarrow H^{0}\left(G_{F_{\mathrm{p}}}, V_{1}\right) \rightarrow H^{0}\left(G_{F_{\mathrm{p}}}, V_{2}\right) \rightarrow H^{0}\left(G_{F_{\mathrm{p}}}, V_{3}\right) \\
& \rightarrow H_{\mathrm{cts} f}^{1}\left(G_{F_{\mathrm{p}}}, V_{1}\right) \rightarrow H_{\mathrm{cts} f}^{1}\left(G_{F_{\mathrm{p}}}, V_{2}\right) \rightarrow H_{\mathrm{cts} f}^{1}\left(G_{F_{\mathrm{p}}}, V_{3}\right) \rightarrow 0 .
\end{aligned}
$$

PROPOSITION 9.6. The graded Lie algebra $\mathrm{Gr}_{\bullet}^{W} \mathfrak{f}_{F, S}$ is free and

$$
H_{\mathrm{cts}}^{1}\left(\mathfrak{f}_{F, S}\right) \cong \bigoplus_{n=1}^{\infty} H_{\mathrm{cts} f}^{1}\left(G_{F, S}, \mathrm{Q}_{\ell}(n)\right) \otimes \mathrm{Q}_{\ell}(-n) \cong \bigoplus_{n=1}^{\infty} \mathrm{Q}_{\ell}(-n)^{d_{n}},
$$

where $d_{n}$ is given as in Theorem 7.2.

Proof. By Theorem 9.5 we have an injection.

$$
H_{\mathrm{cts}}^{2}\left(\mathfrak{f}_{F, S}\right) \hookrightarrow \bigoplus_{n=2}^{\infty} H_{\mathrm{cts}}^{2}\left(G_{F, S}, \mathrm{Q}_{\ell}(n)\right) \otimes \mathrm{Q}_{\ell}(-n) .
$$

Since the right hand side vanishes, $\mathrm{Gr}_{\bullet}^{W} \mathfrak{f}_{F, S}$ is free by Lemma 4.11 . 
The computation of $H_{1}\left(\mathfrak{f}_{F, S}\right)$ follows from Proposition 9.3 and the facts (cf. Example 3.9 in [3, p. 359]) that $H_{\mathrm{cts} f}^{1}\left(G_{F, S}, Q_{\ell}(n)\right)=H_{\mathrm{cts}}^{1}\left(G_{F, S}, Q_{\ell}(n)\right)$ when $n \geqslant 2$ and that there is an inclusion

$$
H_{\mathrm{cts} f}^{1}\left(G_{F, S \cup[\ell]}, \mathrm{Q}_{\ell}(1)\right) \hookrightarrow H_{\mathrm{cts}}^{1}\left(G_{F, S \cup[\ell]}, \mathrm{Q}_{\ell}(1)\right)
$$

corresponding to $\mathcal{O}_{F, S}^{\times} \hookrightarrow \mathcal{O}_{F, S \cup[\ell]}^{\times}$via Kummer characters. Thus,

$$
\operatorname{dim}_{\mathrm{Q}_{\ell}} H_{\mathrm{cts} f}^{1}\left(G_{F, S \cup[\ell]}, \mathrm{Q}_{\ell}(n)\right)=d_{n}
$$

where $d_{n}$ is defined as in Theorem 7.2.

For example, when $\mathcal{O}_{F, S}=\operatorname{Spec} Z, \mathrm{Gr}_{\bullet}^{W} \mathfrak{f}_{F, S}$ is a free Lie algebra generated by $\sigma_{3}, \sigma_{5}, \sigma_{7}, \ldots$

The formula for $\operatorname{Ext}_{\mathcal{M T M}}^{i}\left(\mathcal{O}_{F, S}\right)\left(Q_{\ell}, Q_{\ell}(n)\right)$ in Theorem 9.2 also holds for this case, by replacing $H_{\mathrm{cts}}^{1}\left(G_{F, S}, \mathrm{Q}_{\ell}(1)\right)$ with $H_{\mathrm{cts} f}^{1}\left(G_{F, S}, \mathrm{Q}_{\ell}(1)\right)$.

Remark 9.7. Shiho [32] has announced that his theory of the unipotent crystalline fundamental group implies that the Lie algebra of $Q_{\ell}$-unipotent completion of the fundamental group of a proper smooth curve is crystalline at $\mathfrak{p}$ for any prime above $\ell$ if the curve has a good reduction at $\mathfrak{p}$.

The corresponding result for incomplete varieties, including the projective line minus three points, will follow from the syntomic conjecture for incomplete varieties (cf. [35] for proper case) together with Shiho's methods.

Instead, we established the vanishing of $\sigma_{1}$ using Lemma 8.5. This and the above theorem show conversely that the $Q_{\ell}$-unipotent completion of $\pi_{1}(\mathrm{P}(1 / \mathrm{Q})-$ $\{0,1, \infty\})$ is crystalline, since outer $G_{\ell}$-action factors through $\mathcal{A}_{\mathrm{Q}, \emptyset}$.

THEOREM 9.8. The outer action $G_{\ell} \rightarrow$ Out $\mathcal{P}$ of $G_{\ell}$ on $\ell$-adic unipotent completion of $\pi_{1}(\mathbb{P}(1 / Q)-\{0,1, \infty\})$ is crystalline at $\ell$.

\section{Appendix A. Unipotent Completion}

This appendix is a collection of results needed on unipotent completion. Some of this material may be new, while other results are either folklore or implicit in the literature. Unipotent completion is also known as Malcev completion. One particularly useful approach to unipotent completion is due to Quillen [26, Appendix A].

\section{A.1. UNIPOTENT COMPLETION}

Suppose that $\Gamma$ is a group and that $k$ is a field of characteristic zero. The unipotent completion of $\Gamma$ over $k$ consists of a prounipotent $k$-group $\Gamma_{/ k}^{\mathrm{un}}$ together with a homomorphism $\theta: \Gamma \rightarrow \Gamma_{/ k}^{\mathrm{un}}(k)$. These have the property that every homomorphism $\Gamma \rightarrow U(k)$ from $\Gamma$ to the $k$-rational points of a unipotent $k$-group $U$ factors uniquely through $\theta$. 
Unipotent completion is easily seen to exist:

$$
\Gamma_{/ k}^{\mathrm{un}}=\underbrace{\lim }_{\rho} U_{\rho}
$$

where $\rho$ ranges over all Zariski dense representations $\rho: \Gamma \rightarrow U_{\rho}(k)$ from $\Gamma$ into a unipotent $k$-group.

The universal mapping property implies that unipotent completion is unique up to a canonical isomorphism. The image of $\Gamma$ in its unipotent completion is Zariski dense, since the Zariski closure of the image has the requisite universal mapping property.

Since all finite-dimensional vector spaces are unipotent $k$-groups, there is a canonical isomorphism

$$
H_{1}\left(\Gamma_{/ k}^{\mathrm{un}}(k)\right) \cong H_{1}(\Gamma, k):=\Gamma^{a b} \otimes_{\mathrm{Z}} k,
$$

whenever $H_{1}(\Gamma, k)$ is finite-dimensional.

Unipotent completion behaves well under field extension. The following result is proved in [12].

PROPOSITION A.1. Suppose that $\Gamma$ is a group, that $k$ is a field of characteristic zero and that $H_{1}(\Gamma, k)$ is finite-dimensional. If $K$ is an extension field of $k$, then the natural homomorphism $\Gamma \rightarrow \Gamma_{j k}^{\mathrm{un}}(K)$ induces an isomorphism

$$
\Gamma_{/ K}^{\mathrm{un}} \stackrel{\sim}{\longrightarrow} \Gamma_{/ k}^{\mathrm{un}} \otimes_{k} K .
$$

In particular, if $\Gamma$ is a finitely generated group, then there is a natural isomorphism

$$
\Gamma_{/ k}^{\mathrm{un}} \stackrel{\sim}{\longrightarrow} \Gamma_{/ \mathrm{Q}}^{\mathrm{un}} \otimes_{\mathrm{Q}} k .
$$

The lower central series

$$
\Gamma=L^{1} \Gamma \supseteq L^{2} \Gamma \supseteq L^{3} \Gamma \supset \cdots
$$

of a group $\Gamma$ is defined inductively by

$$
L^{1} \Gamma=\Gamma \text { and } L^{m+1} \Gamma=\left[\Gamma, L^{m} \Gamma\right] .
$$

Define a filtration $D_{k}^{\bullet}$ of $\Gamma$ by

$$
D_{k}^{m} \Gamma=\operatorname{ker}\left\{\Gamma \rightarrow\left(\Gamma_{/ k}^{\mathrm{un}} / L^{m} \Gamma_{/ k}^{\mathrm{un}}\right)(k)\right\} .
$$

This is a central filtration of $\Gamma$, so it follows that $L^{m} \Gamma \subseteq D_{k}^{m} \Gamma$ for all $m$.

THEOREM A.2. If $H_{1}(\Gamma, k)$ is finite-dimensional, then the subgroup $D_{k}^{m} \Gamma$ is the inverse image of the set of torsion elements of $\Gamma / L^{m} \Gamma$ under the quotient mapping $\Gamma \rightarrow \Gamma / L^{m} \Gamma$. The central filtration $D_{k}^{\bullet}$ of $\Gamma$ is the most rapidly descending central series with the property that each $\Gamma / D_{k}^{m} \Gamma$ is a torsion free nilpotent group of length $<m$. If $\Gamma$ is finitely generated, then each $\mathrm{Gr}_{D_{k}}^{m} \Gamma$ is a finitely generated Abelian group, and each $D_{k}^{m} \Gamma / L^{m} \Gamma$ is finite. 
Proof. First recall the elementary fact that the set of torsion elements of a nilpotent group $N$ forms a characteristic subgroup $T$ and that $N / T$ is torsion-free. This implies that the set $R^{m} \Gamma$ of elements of $\Gamma$ that are torsion modulo $L^{m} \Gamma$ is a characteristic subgroup, that $\Gamma / R^{m} \Gamma$ is a torsion-free nilpotent group, and that $R^{m} \Gamma / L^{m} \Gamma$ is a torsion group. It follows that the central series $R^{\bullet} \Gamma$ is the most rapidly descending central series with each $\Gamma / R^{m} \Gamma$ torsion-free.

Let $\mathcal{P}=\Gamma_{/ k}^{\mathrm{un}}$. Since $H_{1}(\Gamma, k)$ is finite-dimensional, each $\mathcal{P} / L^{m} \mathcal{P}$ is unipotent. Since unipotent groups are torsion-free, and since $\Gamma / D_{k}^{m} \Gamma$ is a subgroup of $\mathcal{P}(k) / L^{m} \mathcal{P}(k)$, it follows that $D_{k}^{m} \Gamma \supseteq R^{m} \Gamma$.

It follows from Quillen's version [26, Appendix A] of Malcev's Theorem [21] that there is a unipotent $k$-group $U$ that contains $\Gamma / R^{m} \Gamma$ as a Zariski dense subgroup. The density implies that the length of $U$ is $<m$. The homomorphism $\Gamma \rightarrow U\left(Q_{\ell}\right)$ induces a homomorphism $\mathcal{P} \rightarrow U$ which factors through $\mathcal{P} / L^{m} \mathcal{P}$ as $U$ is unipotent of length $<m$. It follows that $R^{m} \Gamma \supseteq D_{k}^{m} \Gamma$, and that $R^{m} \Gamma=D_{k}^{m} \Gamma$ for all $m$, which implies the result.

\section{A.2. $\ell$-ADIC UNIPOTENT COMPLETION}

There is an obvious variant of unipotent completion for topological groups. Suppose that $\Gamma$ is a topological group and $k$ a topological field with characteristic zero. We shall say that a homomorphism $\Gamma \rightarrow G(k)$ from $\Gamma$ into the $k$-rational points of an algebraic $k$-group $G$ is continuous if it is continuous with respect to the topology on $G(k)$ induced from that of $k$. A homomorphism $\Gamma \rightarrow G(k)$ from $\Gamma$ to the $k$-points of a proalgebraic $k$-group $G$ is continuous if it is the inverse limit of continuous homomorphisms $\Gamma \rightarrow G_{\alpha}(k)$ from $\Gamma$ to each of the finite-dimensional quotients $G_{\alpha}$ of $G$.

The continuous unipotent completion of $\Gamma$ consists of a prounipotent $k$-group $\Gamma_{/ k}^{\mathrm{un}}$ together with a continuous homomorphism $\theta: \Gamma \rightarrow \Gamma_{/ k}^{\mathrm{un}}(k)$. These have the property that every continuous homomorphism $\Gamma \rightarrow U(k)$ from $\Gamma$ to the $k$-rational points of a unipotent $k$-group $U$ factors uniquely through $\theta$.

The continuous unipotent completion of a topological group $\Gamma$ is constructed in much the same way as the standard unipotent completion given above. The universal mapping property of continuous unipotent completion implies that it is unique up to canonical isomorphism.

From now on, we assume that $\Gamma$ is a profinite group and that $k=Q_{\ell}$ with the $\ell$-adic topology. Since compact subgroups of $U\left(Q_{\ell}\right)$ are pro- $\ell$ groups, the $\ell$-adic unipotent completion of $\Gamma$ factors through the pro- $\ell$ completion $\Gamma^{(\ell)}$ of $\Gamma$. Moreover,

$$
\Gamma_{\mathrm{Q}_{\ell}}^{\mathrm{un}} \rightarrow\left(\Gamma^{(\ell)}\right)_{\mathrm{Q}_{\ell}}^{\mathrm{un}}
$$

is an isomorphism since both have the same universal mapping property. Thus, we may assume, without loss of generality, that $\Gamma$ is a pro- $\ell$ group.

The lower central series

$$
\Gamma=L^{1} \Gamma \supseteq L^{2} \Gamma \supseteq L^{3} \Gamma \supset \cdots
$$


of a profinite group $\Gamma$ is defined inductively by

$$
L^{1} \Gamma=\Gamma \text { and } L^{m+1} \Gamma=\text { closure of }\left[\Gamma, L^{m} \Gamma\right] \text { in } \Gamma .
$$

Define a filtration $D_{\ell}^{\bullet}$ of $\Gamma$ by

$$
D_{\ell}^{m} \Gamma=\operatorname{ker}\left\{\Gamma \rightarrow\left(\Gamma_{/ Q_{\ell}}^{\text {un }} / L^{m} \Gamma_{/ Q_{\ell}}^{\text {un }}\right)\left(Q_{\ell}\right)\right\} .
$$

This is a central filtration of $\Gamma$, so it follows that $L^{m} \Gamma \subseteq D_{\ell}^{m} \Gamma$ for all $m$.

THEOREM A.3. If $\Gamma$ is a topologically finitely generated pro- $\ell$ group, then the subgroup $D_{\ell}^{m} \Gamma$ is the inverse image of the set of torsion elements of $\Gamma / L^{m} \Gamma$ under the quotient mapping $\Gamma \rightarrow \Gamma / L^{m} \Gamma$. The central filtration $D_{\ell}^{\bullet}$ of $\Gamma$ is the most rapidly descending central series with the property that each $\Gamma / D_{\ell}^{m} \Gamma$ is a torsion-free nilpotent group of length $<m$. Moreover, each $\mathrm{Gr}_{D_{\ell}}^{m} \Gamma$ is a finitely generated $Z_{\ell}$-module and each $D_{\ell}^{m} \Gamma / L^{m} \Gamma$ is a finite $\ell$-group.

COROLLARY A.4. The $\ell$-adic unipotent completions of $\Gamma / L^{m} \Gamma$ and $\Gamma / D^{m} \Gamma$ are both $\Gamma_{/ Q_{\ell}}^{\text {un }} / L^{m} \Gamma_{/ Q_{\ell}}^{\text {un }}$.

Proof. This follows by a proof that is essentially the same as that of Theorem A.2, taking the continuity of $\Gamma \rightarrow U\left(Q_{\ell}\right)$ into consideration in the current setting. The following lemma is a key ingredient.

LEMMA A.5. If $\Gamma$ is a torsion-free nilpotent pro- $\ell$ group, which is topologically finitely generated, then $\Gamma$ is continuously embeddable in the $Q_{\ell}$-rational points of a unipotent group $U$ over $\mathrm{Q}_{\ell}$.

Proof. The proof is similar to the proof of Proposition 3.6(a) in [26, Appendix A]. Since $\Gamma^{a b}$ is finitely generated, there is a central filtration of $\Gamma$ by closed normal subgroups, each of whose graded quotients is either $Z_{\ell}$ or $Z / \ell$. We prove the lemma by induction on the length of this filtration. There is an exact sequence

$$
1 \rightarrow \Gamma_{1} \rightarrow \Gamma \rightarrow C \rightarrow 1,
$$

where $C$ is $Z_{\ell}$ or $Z_{1} / \ell$. By induction, $\Gamma_{1}$ is embedded in its $\ell$-adic unipotent completion $U_{1}$, which is algebraic by induction. If $C \cong Z_{\ell}$, then $\Gamma \cong C \times \Gamma_{1}$. The conjugacy action of $C$ on $\Gamma_{1}$ lifts by functoriality to a unipotent action on the Lie algebra of $U_{1}$, and thus extends to a unipotent action of $\mathrm{G}_{a}$ on $U_{1}$. It follows that $C \times \Gamma_{1}$ can be continuously embedded in the unipotent group $\mathrm{G}_{a} \times U_{1}$. If $C \cong Z / \ell$, then choose $x \in \Gamma$ whose image generates $C$. Then $x^{\ell} \in U_{1}\left(Q_{\ell}\right)$, and since $U_{1}\left(Q_{\ell}\right)$ is uniquely divisible, there is a unique $x^{\prime} \in U_{1}\left(Q_{\ell}\right)$ with $x^{\ell}=x^{\prime \ell}$. Then $\Gamma$ can be imbedded in $U_{1}\left(Q_{\ell}\right)$ by mapping $x$ to $x^{\prime}$.

\section{A.3. A COMPARISON THEOREM}

There is a close relation between the unipotent completion of a finitely generated group and the $\ell$-adic unipotent completion of its profinite and $\ell$-adic completions. 
THEOREM A.6. If $\Gamma$ is a finitely generated discrete group with pro- $\ell$ completion $\Gamma^{(\ell)}$ and profinite completion $\hat{\Gamma}$, then the three groups

$$
\Gamma_{/ Q}^{\mathrm{un}} \otimes_{\mathrm{Q}} Q_{\ell}, \quad \Gamma_{/ Q_{\ell}}^{(\ell)} \text { and } \quad \hat{\Gamma}_{/ Q_{\ell}}^{\mathrm{un}}
$$

are all canonically isomorphic as prounipotent $\mathrm{Q}_{\ell}$-groups.

The proof reduces to the following result:

LEMMA A.7. Suppose that $\Gamma$ is a finitely generated group with pro- $\ell$ completion $\Gamma^{(\ell)}$. If $U$ is a prounipotent group over $Q_{\ell}$, then every homomorphism $\rho: \Gamma \rightarrow U\left(Q_{\ell}\right)$ is continuous with respect to the pro- $\ell$ topology on $\Gamma$, so that $\rho$ induces a continuous homomorphism $\Gamma^{(\ell)} \rightarrow U\left(\mathrm{Q}_{\ell}\right)$.

Proof. We may assume that $U$ is the upper triangular unipotent subgroup of $G L_{N}\left(Q_{\ell}\right)$ for some $N$. We denote by $U\left(\ell^{m} Z_{\ell}\right)$ the group of matrices whose $i j$ th entry lies in $\ell^{(i-j) m} Z_{\ell}$ when $i>j$, is 1 when $i=j$, and 0 when $i<j$. Since $\Gamma$ is finitely generated, the image of $\rho$ is contained inside $U\left(\ell^{m} Z_{\ell}\right)$ for some $m \in Z$. The filtration

$$
\cdots \supset U\left(\ell^{n} Z_{\ell}\right) \supset U\left(\ell^{n+1} Z_{\ell}\right) \supset \cdots
$$

is a basic set of neighbourhoods of the identity in $U\left(Q_{\ell}\right)$; each quotient is a finitegroup of $\ell$-power order. Since $\Gamma \subseteq U\left(\ell^{m} Z_{\ell}\right)$, the inverse image of each $U\left(\ell^{n} Z_{\ell}\right)$ is a finite index subgroup of $\Gamma$ of $\ell$-power order. The result follows.

Proof of Theorem A.6. It follows from the lemma that $\Gamma \rightarrow \Gamma_{/ Q}^{\mathrm{un}}\left(Q_{\ell}\right)$ is continuous and induces continuous homomorphisms

$$
\hat{\Gamma} \rightarrow \Gamma^{(\ell)} \rightarrow \Gamma_{/ Q}^{\mathrm{un}}\left(Q_{\ell}\right) .
$$

By the universal mapping property of $\ell$-adic unipotent completion, these induce homomorphisms

$$
\hat{\Gamma}_{/ Q_{\ell}}^{\text {un }} \rightarrow \Gamma_{/ Q_{\ell}}^{(\ell)} \rightarrow \Gamma_{/ Q}^{\text {un }} \otimes_{\mathrm{Q}} Q_{\ell} .
$$

But the homomorphism $\Gamma \rightarrow \hat{\Gamma} \rightarrow \hat{\Gamma}_{/ Q_{\ell}}^{\text {un }}\left(Q_{\ell}\right)$ induces a homomorphism $\Gamma_{/ Q}^{\text {un }} \otimes_{Q}$ $Q_{\ell} \rightarrow \hat{\Gamma}_{/ Q_{\ell}}^{\text {un }}$ whose composite with (11) is the canonical isomorphism of Proposition A.1. This completes the proof as the image of $\Gamma^{(\ell)} \rightarrow \Gamma_{/ Q_{\ell}}^{(\ell)}\left(Q_{\ell}\right)$ is Zariski dense.

\section{A.4. AUTOMORPHISMS}

Suppose that $\mathcal{U}$ is a prounipotent group over a field $k$ of characteristic zero with Lie algebra $\mathfrak{u}$. Standard Lie theory implies that there is a natural isomorphism $H_{1}(\mathcal{U}) \cong H_{1}(\mathfrak{l})$. Suppose that this vector space is finite-dimensional over $k$. For each field extension $K$ of $k$, define the group of continuous automorphisms of $\mathcal{U}(K)$ by

$$
\text { Aut } \mathcal{U}(K)=\underset{\leftarrow}{\lim _{m}} \operatorname{Aut}\left(\mathfrak{u} \otimes_{k} K / L^{m} \mathfrak{u} \otimes_{k} K\right)
$$

where $L^{m} \mathfrak{l}$ denotes the $m$ th term of the lower central series of $\mathfrak{u}$. 
PROPOSITION A.8. If $H_{1}(\mathfrak{u})$ is finite-dimensional over $k$, then there is a proalgebraic group $\operatorname{Aut}(\mathcal{U})$ defined over $k$ that represents the functor

$$
K \mapsto \operatorname{Aut}(\mathcal{U}(K))
$$

from field extensions of $k$ to groups. The kernel $\mathcal{K}$ of the natural homomorphism $\mathcal{A u t}(\mathcal{U}) \rightarrow$ Aut $H_{1}(\mathcal{U})$ is prounipotent. The Lie algebra of $\mathcal{A u t} \mathcal{U}$ is isomorphic to Deru, the Lie algebra of continuous derivations of $\mathfrak{u}$.

Proof. Suppose that $\phi$ is an automorphism of $\mathfrak{u}$. Since $H_{1}(\mathfrak{l}) \cong \mathrm{Gr}_{L}^{1} \mathfrak{l t}$, and since the bracket mapping

$$
\mathrm{Gr}_{L}^{n} \mathfrak{u} \otimes \mathrm{Gr}_{L}^{1} \mathfrak{u} \rightarrow \mathrm{Gr}_{L}^{n+1} \mathfrak{u}
$$

is surjective and commutes with $\phi$, we see that $\phi$ acts trivially on $H_{1}(\mathfrak{H})$ if and only if it acts trivially on $\mathrm{Gr}_{L}^{\bullet} \mathfrak{u}$. It follows that the kernel $\mathcal{K}$ of $\mathcal{A u t} \mathfrak{u} \rightarrow$ Aut $H_{1}(\mathfrak{u})$ is a prounipotent group. The last statement follows from standard Lie theory as Aut $\mathcal{U}=$ Aut $\mathfrak{t}$.

Dividing out by the subgroup of inner automorphisms, we obtain:

COROLLARY A.9. If $H_{1}(\mathfrak{u})$ is finite-dimensional over $k$, then there is a proalgebraic group $\mathcal{O}$ ut $(\mathcal{U})$ defined over $k$ that represents the functor

$$
K \mapsto \operatorname{Out}(\mathcal{U}(K))
$$

from field extensions of $k$ to groups. There is a canonical homomorphism $\mathcal{O u t} \mathcal{U} \rightarrow$ Aut $H_{1}(\mathcal{U})$ whose kernel is prounipotent. The Lie algebra of $\mathcal{O}$ ut $\mathcal{U}$ is naturally isomorphic to OutDer $\mathfrak{u}$, the Lie algebra of continuous outer derivations of $\mathfrak{u}$.

COROLLARY A.10. For each finitely generated group $\Gamma$, there are natural homomorphisms

$$
\operatorname{Aut} \Gamma^{(\ell)} \rightarrow \mathcal{A u t}\left(\Gamma_{/ \mathrm{Q}}^{\mathrm{un}} \otimes_{\mathrm{Q}} \mathrm{Q}_{\ell}\right) \rightarrow \mathcal{O} \operatorname{\mathcal {ut}}\left(\Gamma_{/ \mathrm{Q}}^{\mathrm{un}} \otimes_{\mathrm{Q}} Q_{\ell}\right) .
$$

Proof. This follows from Theorem A.6 as there is a natural action of Aut $\Gamma^{(\ell)}$ on $\mathcal{A u t}\left(\Gamma_{/ Q_{\ell}(\ell)}^{\text {un }}\right)$.

From this we recover a result of Nakamura and Takao [25].

COROLLARY A.11. Suppose that $k$ is a subfield of $\mathrm{C}$ and that $X$ is a variety over $k$. Then for each $k$-rational point $x$ of $X$, there is a natural homomorphism

$$
\operatorname{Gal}(\bar{k} / k) \rightarrow \mathcal{A u t} \pi_{1}(X(\mathrm{C}), x)_{/ \mathrm{Q}}^{\mathrm{un}} \otimes \mathrm{Q}_{\ell} .
$$

\section{Appendix B. Continuous Cohomology of Galois Groups}

In this appendix we prove a result, stated below, that gives a computation of the continuous cohomology of the Galois groups needed in this paper. This is well 
known to the experts (cf. [18]), but we have included a proof for the convenience of readers.

\section{B.1. THE RESULT}

Let $F$ be a number field, $\mathcal{O}_{F}$ its ring of integers, $\ell$ a rational prime number, $S$ a finite set of closed points of $\operatorname{Spec} \mathcal{O}_{F}$ containing all the primes of $\mathcal{O}_{F}$ over $\ell$. Let $\mathcal{O}_{F, S}$ be the ring of $S$-integers, so that $\operatorname{Spec} \mathcal{O}_{F, S}=\operatorname{Spec} \mathcal{O}_{F}-S$. Let $G_{F, S}$ denote $\pi_{1}\left(\operatorname{Spec} \mathcal{O}_{F, S}\right.$, Spec $\bar{F}$ ), the Galois group of the maximal algebraic extension of $F$ unramified outside $S$. Let $r_{1}$ and $r_{2}$ be the number of real and imaginary places of $F$, respectively.

The goal of this appendix is to prove the following result.

THEOREM B.1 (Soulé). With notation as above,

$$
\operatorname{dim}_{Q_{\ell}} H_{\mathrm{cts}}^{1}\left(G_{F, S}, Q_{\ell}(n)\right)= \begin{cases}r_{1}+r_{2}+\# S-1, & \text { if } n=1, \\ r_{1}+r_{2}, & \text { if } n \text { is odd and } \geqslant 3, \\ r_{2}, & \text { if } n \text { is even and positive. }\end{cases}
$$

In addition, $H_{\mathrm{cts}}^{2}\left(G_{F, S}, Q_{\ell}(n)\right)$ vanishes for all $n \geqslant 2$.

The continuous cohomology $H_{\mathrm{cts}}^{\bullet}$ is the one defined in [34, Sect. 2]. We reduce the proof to the computation of the étale cohomology of $\operatorname{Spec} \mathcal{O}_{F, S}$ by the following proposition.

PROPOSITION B.2. For $i=1,2$ and all $n \in Z$, there is a natural isomorphism

$$
H_{\mathrm{cts}}^{i}\left(G_{F, S}, \mathrm{Q}_{\ell}(n)\right) \stackrel{\simeq}{\longrightarrow} H_{\mathrm{et}}^{i}\left(\operatorname{Spec} \mathcal{O}_{F, S}, \mathrm{Q}_{\ell}(n)\right) .
$$

In étale cohomology we have (cf. [23]) a canonical isomorphism

$$
H_{e t}^{i}\left(\operatorname{Spec} \mathcal{O}_{F, S}, Q_{\ell}(n)\right) \cong\left[\overleftarrow{m}_{m} H_{\mathrm{et}}^{i}\left(\operatorname{Spec} \mathcal{O}_{F, S}, Z_{\ell}(n) / \ell^{m}\right)\right] \otimes_{\mathrm{Z}_{\ell}} Q_{\ell} .
$$

(For an Abelian group $M$, let $M / \ell^{m}=M / \ell^{m} M$ and denote the $\ell^{m}$-torsion of $M$ by $M\left[\ell^{m}\right]$.) Since the canonical map

$$
H_{\mathrm{cts}}^{i}\left(G_{F, S}, Z_{\ell}(n) / \ell^{m}\right) \rightarrow H_{\mathrm{e} t}^{i}\left(\operatorname{Spec} \mathcal{O}_{F, S}, Z_{\ell}(n) / \ell^{m}\right)(i \geqslant 1)
$$

is isomorphic [24, Proposition 2.9], Proposition 11.2 is a consequence of the following lemma.

LEMMA B.3. When $i=1,2$, there is a natural isomorphism

$$
\left.H_{\mathrm{cts}}^{i}\left(G_{F, S}, \mathrm{Q}_{\ell}(n)\right) \cong \underset{\stackrel{[}{m}}{\lim } H_{\mathrm{cts}}^{i}\left(G_{F, S}, Z_{\ell}(n) / \ell^{m}\right)\right] \otimes_{\mathrm{Z}_{\ell}} Q_{\ell} .
$$

Note that $H_{\mathrm{cts}}^{\bullet}\left(G, Z_{\ell}(n) / \ell^{m}\right)$ is the usual cohomology of profinite groups defined, for example, in [29]. 
Proof. The short exact sequence

$$
0 \rightarrow Z_{\ell}(n) \rightarrow Q_{\ell}(n) \rightarrow\left(Q_{\ell} / Z_{\ell}\right)(n) \rightarrow 0
$$

gives a long exact sequence [34, p. 259]

$$
\begin{aligned}
\cdots \rightarrow H_{\mathrm{cts}}^{i}\left(G_{F, S}, Z_{\ell}(n)\right) & \rightarrow H_{\mathrm{cts}}^{i}\left(G_{F, S}, \mathrm{Q}_{\ell}(n)\right) \\
& \rightarrow H_{\mathrm{cts}}^{i}\left(G_{F, S},\left(Q_{\ell} / Z_{\ell}\right)(n)\right) \rightarrow H_{\mathrm{cts}}^{i+1}\left(G_{F, S}, Z_{\ell}(n)\right) \rightarrow \cdots
\end{aligned}
$$

Since $G_{F, S}$ is compact, the proof of $[34,2.3]$ shows that $H_{\mathrm{cts}}^{i}\left(G_{F, S},\left(\mathrm{Q}_{\ell} / Z_{\ell}\right)(n)\right)$ is torsion. So, after tensoring with $Q_{\ell}$, we have an isomorphism

$$
H_{\mathrm{cts}}^{i}\left(G_{F, S}, Q_{\ell}(n)\right) \cong H_{\mathrm{cts}}^{i}\left(G_{F, S}, Z_{\ell}(n)\right) \otimes_{Z_{\ell}} Q_{\ell}
$$

When $i=0,1, H_{\mathrm{cts}}^{i}\left(G_{F, S}, Z_{\ell}(n) / \ell^{m}\right)$ is finite. This is obvious when $i=0$. When $i=1$ it follows from class field theory. Indeed, reduce to the case where $Z_{\ell}(n) / \ell^{m}$ has trivial Galois action by passing to a finite extension. Then $H_{\mathrm{cts}}^{1}\left(G_{F, S}, Z_{\ell}(n) / \ell^{m}\right)$ is $\operatorname{Hom}^{\mathrm{cts}}\left(G_{F, S}, Z_{\ell} / \ell^{m}\right)$, which is finite. The corollary to [34, Prop. 2.2] then implies that

$$
H_{\mathrm{cts}}^{i}\left(G_{F, S}, Z_{\ell}(n)\right)=\underset{m}{\lim } H_{\mathrm{cts}}^{i}\left(G_{F, S}, Z_{\ell \ell}(n) / \ell^{m}\right) .
$$

Tensoring with $Q_{\ell}$ completes the proof.

B.2. Proof of Theorem B.1. First, we dispense with the case where $n=1$ by showing that

$$
\operatorname{dim}_{Q_{\ell}} H_{\text {ét }}^{1}\left(\operatorname{Spec} \mathcal{O}_{F, S}, Q_{\ell}(1)\right)=r_{1}+r_{2}+\# S-1 .
$$

Take the projective limit over $m$ of the Kummer sequence [23, p. 128]

$$
\begin{aligned}
0 \rightarrow H_{\mathrm{et}}^{0}\left(\operatorname{Spec} \mathcal{O}_{F, S}, \mathbb{G}_{m}\right) / \ell^{m} & \rightarrow H_{\mathrm{e} t}^{1}\left(\operatorname{Spec} \mathcal{O}_{F, S}, \mu_{\ell^{m}}\right) \\
& \rightarrow H_{\mathrm{e} t}^{1}\left(\operatorname{Spec} \mathcal{O}_{F, S}, \mathrm{G}_{m}\right)\left[\ell^{m}\right] \rightarrow 0 .
\end{aligned}
$$

The limit of the center term is $H_{\text {ét }}^{1}\left(\operatorname{Spec} \mathcal{O}_{F, S}, Z_{\ell}(1)\right)$. Since the ideal class group $H_{\text {et }}^{1}\left(\operatorname{Spec} \mathcal{O}_{F, S}, \mathbb{G}_{m}\right)$ is finite and the maps in the inverse system are multiplication by powers of $\ell$, the limit of the right hand term vanishes. It follows that the limits of the first two terms are isomorphic. Assertion (12) now follows as $\mathcal{O}_{F, S}^{\times}$has rank $r_{1}+r_{2}+\# S-1$ by the Dirichlet Unit Theorem.

To address the case where $n>1$, we need the following result of Soule [33, p. 376].

THEOREM B.4 [Soulé]. If $F$ is a number field and $\ell$ an odd prime number, then

$$
\operatorname{dim}_{Q_{\ell}} H_{\mathrm{et}}^{1}\left(\operatorname{Spec} \mathcal{O}_{F}[1 / \ell], Q_{\ell}(n)\right)= \begin{cases}r_{1}+r_{2}, & \text { if } n \text { is odd and } \geqslant 3, \\ r_{2}, & \text { if } n \text { is even and positive. }\end{cases}
$$

In addition, $H_{\mathrm{ett}}^{2}\left(\operatorname{Spec} \mathcal{O}_{F}[1 / \ell], Q_{\ell}(n)\right)$ vanishes for all $n \geqslant 2$. 
This theorem is also true when $\ell=2$, following from [27]. An explicit proof for the case of $F=Q$ has been written down by Sharifi [31].

Theorem B.1 follows directly from this computation when $S$ is the set of primes lying over $\ell$. For the general case, it is enough to prove the following statement (cf. one may use [18, Eqn. 13]).

LEMMA B.5. If $z$ is a closed point of $\operatorname{Spec} \mathcal{O}_{F, S}$ and $S^{\prime}=S \cup\{z\}$, then

$$
H_{\mathrm{et}}^{i}\left(\operatorname{Spec} \mathcal{O}_{F, S}, Q_{\ell}(n)\right) \cong H_{\mathrm{et}}^{i}\left(\operatorname{Spec} \mathcal{O}_{F, S^{\prime}}, \mathrm{Q}_{\ell}(n)\right)
$$

for all $i \geqslant 0$ provided $n \neq 0,1$.

Proof. Let $X=\operatorname{Spec} \mathcal{O}_{F, S}$ and $U=\operatorname{Spec} \mathcal{O}_{F, S^{\prime}}$. By [23, p. 92, Prop. 1.25] there is a long exact sequence

$$
\cdots \rightarrow H_{z}^{i}\left(X, \mathrm{Q}_{\ell}(n)\right) \rightarrow H_{\mathrm{et}}^{i}\left(X, \mathrm{Q}_{\ell}(n)\right) \rightarrow H_{\mathrm{et}}^{i}\left(U, \mathrm{Q}_{\ell}(n)\right) \rightarrow H_{z}^{i+1}\left(X, \mathrm{Q}_{\ell}(n)\right) \rightarrow \cdots
$$

where $H_{z}^{\bullet}$ denotes étale cohomology with support on $z$. Thus it is enough to show that $H_{z}^{i}\left(X, Q_{\ell}(n)\right)$ vanishes for all $i$ unless $n=0$ or 1 .

Let $\mathcal{X}$ denote the Henselization of $\operatorname{Spec} \mathcal{O}_{F}$ at $z$. Then by [23, p. 93 Cor. 1.28] we have

$$
H_{z}^{i}\left(X, \mathrm{Q}_{\ell}(n)\right) \cong H_{z}^{i}\left(\mathcal{X}, \mathrm{Q}_{\ell}(n)\right)
$$

so it suffices to establish the vanishing of the right-hand side.

Set $\mathcal{U}=\mathcal{X}-z$ and note that this is just $\operatorname{Spec} K(\mathcal{X})$. By a second application of the long exact sequence [23, p. 92, Prop. 1.25] applied to $(\mathcal{X}, \mathcal{U})$, we see that if $H_{\mathrm{et}}^{i}\left(\mathcal{X}, \mathrm{Q}_{\ell}(n)\right)$ and $H_{\mathrm{et}}^{i}\left(\mathcal{U}, \mathrm{Q}_{\ell}(n)\right)$ both vanish when $n \neq 0,1$, then $H_{z}^{i}\left(\mathcal{X}, \mathrm{Q}_{\ell}(n)\right)$ vanishes.

Firstly, by [23, p. 224, Cor. 2.7], we have

$$
H_{\mathrm{et}}^{i}\left(\mathcal{X}, \mathrm{Q}_{\ell}(n)\right)=H_{\mathrm{et}}^{i}\left(z, Q_{\ell}(n)\right)=H_{\mathrm{cts}}^{i}\left(\hat{\mathrm{Z}}, \mathrm{Z}_{\ell}(n)\right) \otimes Q_{\ell} .
$$

As in Example 4.12, the right-hand group is trivial unless $n=0$.

Set $\mathcal{U}^{\text {ur }}=\operatorname{Spec} F(\mathcal{X})$, where $F(\mathcal{X})$ is the maximal algebraic extension of $K(\mathcal{X})$ unramified at $z$. To prove the second vanishing we consider the Hochshild-Serre spectral sequence (cf. [23, p. 105, Thm. 2.20])

$$
H_{\mathrm{et}}^{a}\left(z, H_{\mathrm{et}}^{b}\left(\mathcal{U}^{\mathrm{ur}}, \mathrm{Q}_{\ell}(n)\right)\right) \Rightarrow H_{\mathrm{et}}^{a+b}\left(\mathcal{U}, \mathrm{Q}_{\ell}(n)\right) .
$$

According to [29], $\mathcal{U}^{\text {ur }}$ has cohomological dimension at most 1 , so that

$$
H_{\mathrm{ett}}^{b}\left(\mathcal{U}^{\mathrm{ur}}, Q_{\ell}(n)\right)=0 \text { when } b>1 .
$$

Since the pro- $\ell$ Abelianization of the inertia group $\pi_{1}\left(\mathcal{U}^{\text {ur }}\right)$ is $Z_{l}(1)$ as a Galois module, we have

$$
H_{\mathrm{et}}^{1}\left(\mathcal{U}^{\mathrm{ur}}, \mathrm{Q}_{\ell}(n)\right)=\operatorname{Hom}^{\mathrm{cts}}\left(\pi_{1}\left(\mathcal{U}^{\mathrm{ur}}\right), \mathrm{Q}_{\ell}(n)\right) \cong \mathrm{Q}_{\ell}(n-1) .
$$

The required vanishing follows by plugging this, and the fact that $H_{\mathrm{et}}^{0}\left(\mathcal{U}^{\mathrm{ur}}, Q_{\ell}(n)\right)$ is $Q_{\ell}(n)$, into the spectral sequence. 


\section{Acknowledgements}

It is a pleasure to thank all those with whom we have had helpful discussions: Akio Tamagawa, Masato Kurihara and Chad Schoen for discussions on étale cohomology, Kazuya Kato and Takeshi Tsuji for discussions on crystalline representations, and Yasutaka Ihara for his generous comments on and clarification about the origin and history of the conjectures considered in this paper. We would also like to thank Alexandre Goncharov, for pointing out the relevance of the work of Beilinson and Deligne [1], Romyar Sharifi, for useful comments on and corrections to the manuscript, and the referee who made many useful comments.

R. H. was supported in part by grants from the National Science Foundation. M. M. was supported in part by a Mombusho Grant and also by MSRI during a visit in the fall of 1999 .

\section{References}

1. Beilinson, A. and Deligne, P.: Motivic polylogarithms and Zagier's conjecture, unpublished manuscript, 1992.

2. Borel, A.: Linear Algebraic Groups, 2nd edn., Grad. Texts in Math. 126, Springer-Verlag, New York, 1991.

3. Bloch, S. and Kato, K.: L-functions and Tamagawa numbers of motives, The Grothendieck Festschrift Volume I, Progr. in Math. 86, Birkhäuser, 1990, pp. 333-400.

4. Deligne, P.: Théorie de Hodge II, Inst. Hautes Études Sci. Publ. Math. 40, (1971), 5-57.

5. Deligne, P.: Le groupe fondamental de la droite projective moins trois points, In: Galois Groups over Q (Berkeley, CA, 1987), Math. Sci. Res. Inst. Publ., 16, Springer, New York Berlin, 1989, pp. 79-297.

6. Deligne, P. and Milne, J.: Tannakian categories, In: P. Deligne, J. Milne, A. Ogus and K.-Y. Shih (eds), Hodge Cycles, Motives, and Shimura Varieties, Lecture Notes in Math. 900, Springer-Verlag, New York, 1982.

7. Drinfeld, V.: On quasitriangular quasi-Hopf algebras and on a group that is closely connected with $\mathrm{Ga} \overline{\mathrm{Q}} / \mathrm{Q}$ ) (Russian) Algebra i Analiz 2 (1990), 149-181; translation in Leningrad Math. J. 2(4) (1991), 829-860.

8. Fontaine, J. M.: Sur certains types de représentations $p$-adiques du groupe de Galois d'un corps local: construction d'un anneau de Barsotti-Tate, Ann. of Math. 115 (1982), 529-577.

9. Grothendieck, A.: Revêtement étales et groupe fondamental (SGA 1), Lecture Notes in Math. 224, Springer-Verlag, New York, 1971.

10. Goncharov, A.: Volumes of hyperbolic manifolds and mixed Tate motives, J. Amer. Math. Soc. 12 (1999), 569-618.

11. Goncharov, A.: Multiple $\zeta$-values, Galois groups, and geometry of modular varieties, European Congress of Mathematics Vol. I (Barcelona, 2000), Progr. Math., 201, Birkhäuser, Basel, 2001, pp. 361-392.

12. Hain, R.: Completions of mapping class groups and the cycle $C-C^{-}$, In: C.-F. Bödigheimer and R. Hain, (eds), Mapping Class Groups and Moduli Spaces of Riemann Surfaces, Contemp. Math. 150 (1993), 75-105.

13. Hain, R.: Hodge-de Rham theory of relative Malcev completion, Ann. Sci. École Norm. Sup. 31 (1998), 47-92.

14. Hain, R. and Matsumoto, M.: Tannakian fundamental groups associated to Galois groups, In: Galois groups and Fundamental Groups, Leila Schneps (ed.), MSRI Publ. 41, Cambridge Univ. Press, 2003, pp. 183-216. 
15. Ihara, Y.: Profinite braid groups, Galois representations and complex multiplications, Ann. of Math. 123 (1986), 43-106.

16. Ihara, Y.: The Galois representation arising from $\mathbb{P}^{1}-\{0,1, \infty\}$ and Tate twists of even degree, In: Galois Groups over Q, Publ. MSRI, 16, Springer-Verlag, New York, 1989, pp. 299-313.

17. Ihara, Y.: Some arithmetic aspects of Galois actions in the pro- $p$ fundamental group of $\mathrm{P}^{1}-\{0,1, \infty\}$, Arithmetic Fundamental Groups and Noncommutative Algebra (Berkeley, $C A$, 1999), Proc. Sympos, Pure Math. 70, Amer. Math. Soc., Providence, 2002, pp. 247-273.

18. Jannsen, U.: On the $\ell$-adic cohomology of varieties over number fields and its Galois cohomology, In: Galois Groups over Q, Publ. MSRI, 16, Springer-Verlag, New York, 1989, pp. 315-360.

19. Jantzen, J. C.: Representations of Algebraic Groups, Pure Appl. Math. 131, Academic Press, New York, 1987.

20. Levine, M.: Mixed Motives, Math. Surveys Monogr., 57, Amer. Math. Soc., Providence, 1998.

21. Malcev, A.: Nilpotent torsion-free groups, (Russian) Izvestiya Akad. Nauk. SSSR. Ser. Mat. 13 (1949), 201-212.

22. Matsumoto, M.: On the Galois image in derivation of $\pi_{1}$ of the projective line minus three points, In: Recent Developments in the Inverse Galois Problem (Seattle, WA, 1993), Contemp. Math. 186 (1995), 201-213.

23. Milne, J. S.: Étale Cohomology, Princeton Math. Ser. 33, Princeton Univ. Press, 1980.

24. Milne, J. S.: Arithmetic Duality Theorems, Perspect. Math. 1, Academic Press, New York, 1986.

25. Nakamura, H. and Takao, N.; Galois rigidity of pro-l pure braid groups of algebraic curves, Trans. Amer. Math. Soc. 350 (1998), 1079-1102.

26. Quillen, D.: Rational homotopy theory, Ann. Math. 90 (1969), 205-295.

27. Rognes J. and Weibel, C.: Two-primary algebraic $K$-theory of rings of integers in number fields, (Appendix A by Manfred Kolster), J. Amer. Math. Soc. 13 (2000), 1-54.

28. Serre, J.-P.: Local Fields, Translated from the French by Marvin Jay Greenberg, Grad. Texts in Math. 67, Springer-Verlag, New York, 1979.

29. Serre, J.-P.: Cohomologie galoisienne, Lecture Notes in Math. 5, Heidelberg, 1964. English translation: Galois Cohomology, Springer-Verlag, Berlin, 1997.

30. Serre, J.-P.: Lie Algebras and Lie Groups, 1964 Lectures given at Harvard University, 2nd edn, Lecture Notes in Math., 1500, Springer-Verlag, Berlin, 1992.

31. Sharifi, R.: Letter to Matsumoto, April 28, 2000.

32. Shiho, A.: Fundamental groups, homotopy groups and p-adic Hodge Theory, in preparation.

33. Soulé, C.: On higher-p-adic regulators, In: Lecture Notes in Math. 854, Springer-Verlag, Berlin, 1981, pp. 372-401.

34. Tate, J.: Relations between $K_{2}$ and Galois cohomology, Invent. Math. 30 (1976), $257-274$.

35. Tsuji, T.: p-adic étale cohomology and crystalline cohomology in the semi-stable reduction case, Invent. Math. 137 (1999), 233-411.

36. Tsunogai, H.: On ranks of the stable derivation algebra and Deligne's problem, Proc. Japan Acad. Ser. A 73 (1997), 29-31.

37. Voevodsky, V., Suslin, A. and Friedlander, E.: Cycles, Transfers, and Motivic Homology Theories, Ann. Math. Stud. 143, Princeton Univ. Press, 2000.

38. Yoneda, N.: On Ext and exact sequences, J. Fac. Sci. Univ. Tokyo Sect. I 8 (1960), $507-576$. 